nephron

Practice

\title{
UK Renal Registry 17th Annual Report: Chapter 102013 Multisite Dialysis Access Audit in England, Northern Ireland and Wales and 2012 PD One Year Follow-up: National and Centre-specific Analyses
}

\author{
Anirudh Rao ${ }^{a}$, David Pitcher ${ }^{a}$, Richard Fluck ${ }^{b}$, Mick Kumwenda ${ }^{c}$ \\ ${ }^{a}$ UK Renal Registry, Bristol, UK; ${ }^{b}$ Derby Hospitals Foundation Trust, UK; ' Glan Clwyd Hospital, Rhyl, UK
}

\section{Key Words}

Chronic kidney disease - Diabetes . Dialysis . End stage renal disease - Established renal failure - Haemodialysis . Peritoneal dialysis - Prevalence $\cdot$ Renal replacement therapy - Transplantation - Treatment modality - Vascular access

\begin{abstract}
Background: Dialysis access should be timely, minimise complications and maintain functionality. The aim of the second combined vascular and peritoneal dialysis access audit was to examine practice patterns with respect to dialysis access and highlight variations in practice between renal centres. Methods: The UK Renal Registry collected centre-specific information on incident vascular and peritoneal dialysis access outcome measures in patients from England, Wales and Northern Ireland (EW\&NI), including patient demographics, dialysis access type (at start of
\end{abstract}

dialysis and three months after start of dialysis), surgical assessment and access functionality. Centres who had reported data on incident PD patients for the previous 2012 audit were additionally asked to provide one year follow up data for this group. The findings were compared to the audit measures stated in Renal Association clinical practice guidelines for dialysis access. Results: Fifty-seven centres in EW\&NI (representing 92\% of all centres) returned data on first access from 3,663 incident HD patients and 1,022 incident PD patients. A strong relationship was seen between surgical assessment and the likelihood of starting HD with an arteriovenous fistula (AVF). Twenty-four centres were at least two standard deviations below the $65 \%$ target for incident patients starting haemodialysis on AVF and only eight centres (14\%) were within two standard deviations of the $85 \%$ target for prevalent haemodialysis patients on AVF. Conclusions: There was wide practice variation across the UK in provision of both HD and PD access which requires further exploration. 


\section{Introduction}

The second combined vascular and peritoneal dialysis access audit in England, Wales and Northern Ireland represents the findings from the 2013 data collection period for patients starting dialysis between 1st January 2013 and 31st December 2013. Previously, vascular and peritoneal dialysis access audits have been published separately $[1,2]$. The combined access audit provides information on timely and appropriate access interventions in order to achieve permanent access based on the recommendations and quality requirements stated in Renal Association clinical practice guidelines and Vascular Access guidelines for Haemodialysis and Peritoneal access $[3,4]$. The core principal of these audits has been to highlight the performance variation of renal centres across England, Wales and Northern Ireland and explore factors that may contribute to the provision of excellent quality vascular and peritoneal access.

The term established renal failure used within this chapter is synonymous with the terms end stage renal failure and end stage kidney disease, which are in more widespread international usage. Patients have disliked the term 'end stage' which reflects the inevitable outcome of this disease.

\section{Methods}

All adult renal centres in England, Wales and Northern Ireland were contacted regarding vascular and peritoneal access for all incident and prevalent dialysis patients (centre level only) in 2013. Data were collected using Microsoft Excel spreadsheets circulated by the UK Renal Registry (UKRR). Of 62 centres contacted, data were received from 57 centres. Definitions were refined from the previous audit performed in 2013 based on the quality of the returned questionnaires and the feedback received from centres.

Patients who were identified by the renal centres as having acute kidney injury (AKI) in the free text fields or patients who were reported to have recovered renal function within three months were categorised as having AKI for the purposes of this audit and excluded $(n=367 / 5,105)$. The remaining records received were validated against the UKRR database to confirm that the population collected at each centre for the audit was the same as, or representative of, the incident population at that centre as collected via the usual UKRR quarterly return. Data checks were made by cross-referencing with the UKRR database. Any patients identified from the UKRR as not incident to dialysis between 1st January 2013 and 31st December 2013 were excluded. Similarly, where the reported prevalent numbers from the audit did not match those in the UKRR database, those centres were excluded. The cross-referencing also enabled ascertainment of information on mortality within three months of commencing dialysis.

Centres who reported data on peritoneal dialysis (PD) patients in the 2012 vascular and peritoneal access audit were asked to complete a one year follow up of their PD patients. Additional information was requested on the date of PD catheter failure, the reason for catheter failure, the number of catheters used during the year, and the modality in use at one year after starting PD.

Table 10.1 lists the summary of audit measures as stated in the Renal Association clinical practice guidelines, with explanation for why some of the audit measures were not reported.

Patients starting haemodialysis (HD) were grouped by type of first vascular access: arteriovenous fistula (AVF), arteriovenous graft (AVG), tunnelled dialysis line (TL), non-tunnelled dialysis line (NTL). Patients starting PD were categorised by the insertion technique: laparoscopic, peritoneoscopic, open surgery, percutaneous. Access at three months was defined as the type of access in use at three months after starting dialysis. If a patient was no longer receiving dialysis at three months then the reason was recorded instead, for example died or transplanted. Referral time was defined as the number of days between the date of first being seen by a renal physician and the date of commencing dialysis. Patients were classified as presenting late if they had a referral time of less than 90 days. In the analyses involving whether or not a patient had received surgical assessment at least three months before starting dialysis, patients were excluded if they were categorised as a late presenter.

Access failure was defined as the access no longer being usable for dialysis. Data about the date and cause of access failure were collected. Access failure was censored for death, transplantation, withdrawal from renal replacement therapy (RRT) and elective switching of access type. It was the intention to only capture access failures relating to the first type of access. If the reason recorded for access failure was incompatible with the first type of access recorded then the data was not included in this analysis.

Separate or combined analyses have been performed for incident HD patients and incident PD patients as appropriate. Due to the exploratory nature of the audit the analyses have been limited to descriptive statistics of frequencies, percentages and unadjusted associations between variables. If a centre had more than $50 \%$ missing returns for a particular data field, then all patients from the centre were excluded from analyses involving that data field. The data were analysed using SAS 9.3.

\section{Results}

\section{Data completeness}

Fifty-seven centres returned data on first dialysis access for 3,663 incident HD patients and 1,022 incident PD patients. The UKRR incident patient data for the same year were 4,030 $\mathrm{HD}$ and 1,108 PD, thus there were access returns on $91 \%$ of $\mathrm{HD}$ and $92 \%$ of PD patients.

Seventeen patients were excluded from all analyses due to missing RRT start date or first access type. Figure 10.1 illustrates the data completeness for key variables. 
Table 10.1. Summary of audit measures stated in Renal Association clinical practice guidelines for dialysis access

RA audit measure $\quad$ Reported Reason for non-inclusion

\section{HD Access}

1 Proportion of patients whose first haemodialysis treatment is with an arteriovenous fistula

1a Stratified by new patients with established renal failure and known to the nephrology Yes team for $>90$ days

1b Stratified by new patients with established renal failure and known to the nephrology Yes team for $\leq 90$ days

1c Patients with a failed renal transplant

1d Patients transferred permanently from PD to haemodialysis

$265 \%$ of all patients commencing haemodialysis should commence with an AV fistula

3 A centre should measure the proportion of prevalent long term haemodialysis patients receiving dialysis via a fistula, an arteriovenous graft and a tunnelled or a nontunnelled line

$4 \quad 85 \%$ of all prevalent patients on haemodialysis should receive dialysis via a functioning arteriovenous fistula

5 Complications related to vascular access

5a Rupture of vascular access (fistula and graft)

Yes
Yes
Yes
No $\quad$ Not captured by the audit
No $\quad$ Not captured by the aud
Yes
Yes
Yes
Yes
Partly Incident patients only

\section{PD access}

1 Catheter patency - more than $80 \%$ of catheters should be patent at 1 year (censoring for death and elective modality change)

2 Complications following PD catheter insertion

2a Bowel perforation $<1 \%$

$2 \mathrm{~b}$ Significant haemorrhage $<1 \%$

2c Exit site infection within 2 weeks of catheter insertion $<5 \%$

$2 \mathrm{~d}$ Peritonitis within 2 weeks of catheter insertion $<5 \%$

2e Functional catheter problem requiring manipulation or replacement or leading to technique failure $<20 \%$

\section{Yes}

Yes

No Not captured by the audit No Not captured by the audit No Not captured by the audit Yes

No Not captured by the audit

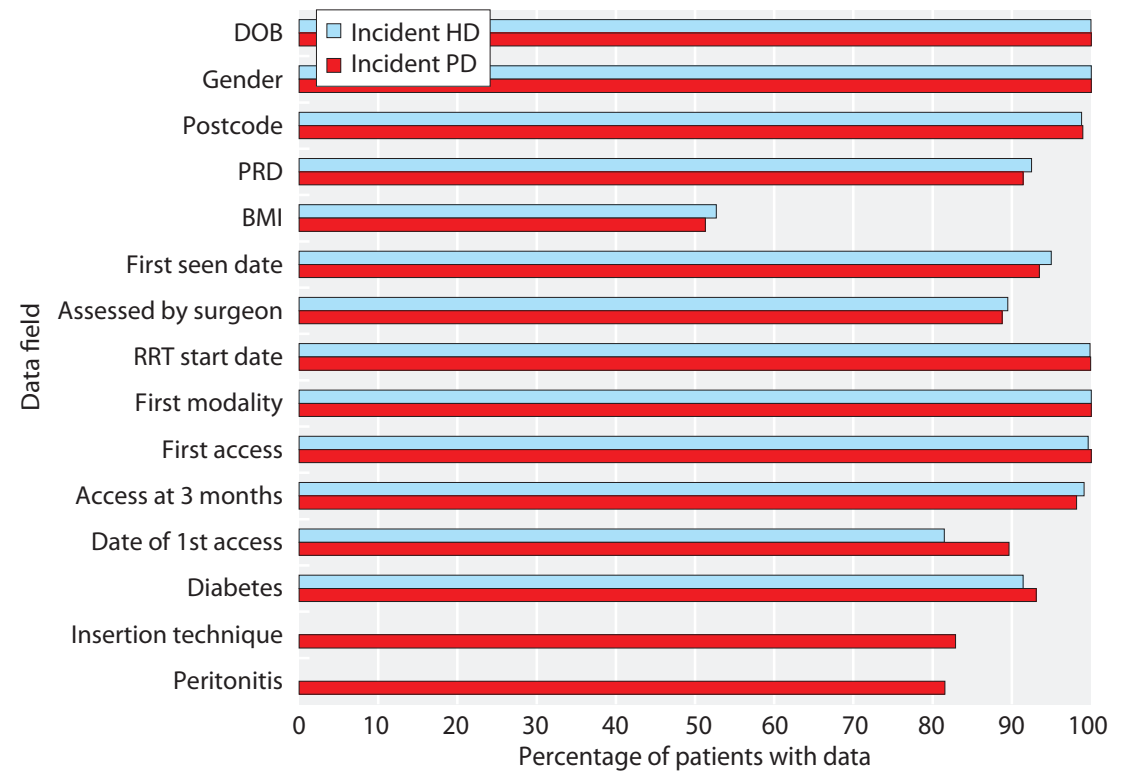

Fig. 10.1. Data completeness for key variables, stratified by first modality $\mathrm{DOB}=$ date of birth; $\mathrm{PRD}=$ primary renal diagnosis; $\mathrm{BMI}=$ body mass index 
Table 10.2. Patient demographics

\begin{tabular}{|c|c|c|c|c|}
\hline Variable & & $\begin{array}{c}\text { Total } \\
N=4,668\end{array}$ & $\begin{array}{c}\mathrm{HD} \\
N=3,647\end{array}$ & $\begin{array}{c}\mathrm{PD} \\
N=1,021\end{array}$ \\
\hline Age & Median (IQR) & $66(53,76)$ & $67(55,76)$ & $60(47,71)$ \\
\hline BMI & Median (IQR) & $27(24,32)$ & $28(24,32)$ & $27(24,31)$ \\
\hline Gender & $\begin{array}{l}\text { Female } \\
\text { Male }\end{array}$ & $\begin{array}{c}N(\%) \\
1,663(35.6) \\
3,005(64.4)\end{array}$ & $\begin{array}{c}N(\%) \\
1,306(35.8) \\
2,341(64.2)\end{array}$ & $\begin{array}{c}N(\%) \\
357(35) \\
664(65)\end{array}$ \\
\hline Diabetes & $\begin{array}{l}\text { Missing } \\
\text { Yes } \\
\text { No }\end{array}$ & $\begin{array}{c}372(8) \\
2,677(57.3) \\
1,619(34.7)\end{array}$ & $\begin{array}{cl}301 & (8.3) \\
2,031 & (55.7) \\
1,315 & (36.1)\end{array}$ & $\begin{array}{c}71(7) \\
646(63.3) \\
304(29.8)\end{array}$ \\
\hline PRD & $\begin{array}{l}\text { Missing } \\
\text { Diabetes } \\
\text { Glomerulonephritis } \\
\text { Hypertension } \\
\text { Other } \\
\text { Polycystic kidney } \\
\text { Pyelonephritis } \\
\text { Renal vascular disease } \\
\text { Uncert }\end{array}$ & $\begin{aligned} 353 & (7.6) \\
1,218 & (26.1) \\
598 & (12.8) \\
368 & (7.9) \\
673 & (14.4) \\
286 & (6.1) \\
247 & (5.3) \\
264 & (5.7) \\
661 & (14.2)\end{aligned}$ & $\begin{array}{l}265(7.3) \\
980(26.9) \\
430(11.8) \\
291(8) \\
572(15.7) \\
184(5) \\
183(5) \\
225(6.2) \\
517(14.2)\end{array}$ & $\begin{aligned} 88 & (8.6) \\
238 & (23.3) \\
168 & (16.5) \\
77 & (7.5) \\
101 & (9.9) \\
102 & (10) \\
64 & (6.3) \\
39 & (3.8) \\
144 & (14.1)\end{aligned}$ \\
\hline
\end{tabular}

$\mathrm{IQR}=$ interquartile range; $\mathrm{BMI}=$ body mass index; $\mathrm{PRD}=$ primary renal diagnosis; $\mathrm{HD}=$ haemodialysis; $\mathrm{PD}=$ peritoneal dialysis

Variations in first dialysis access

Patient demographics

The median patient age when starting RRT was 67 years in the HD cohort and 60 years for patients commencing PD. Overall, $64.4 \%$ of the patients were male, $35.6 \%$ female; the proportional distribution of the sexes was similar for both the HD and PD subgroups.

A significant proportion of patients starting dialysis had diabetes (57.3\%), however, diabetes associated nephropathy was the primary renal disease (PRD) in only $26.1 \%$ (table 10.2 ).

Table 10.3 presents $\mathrm{HD}$ and $\mathrm{PD}$ patient subgroups stratified by age, dichotomised body mass index (BMI) $(\leqslant 30$ or $>30)$, PRD, referral time $(<90$ or $\geqslant 90$ days) and surgical assessment status.

There was an association between the access modality ( $\mathrm{HD}$ vs. $\mathrm{PD}$ ), referral time ( $<90$ days vs. $\geqslant 90$ days) and surgical assessment status in excess of three months prior to dialysis start. The following observations can be made:

For HD:

- AVF was the initial access for $40.7 \%$ of patients, with $0.9 \%$ on an AVG, $37.2 \%$ on a tunnelled line and $21.3 \%$ on a non-tunnelled line. The percentage of patients starting with an AVF has been stable for the last three years with the majority of centres failing to achieve the target as stated in the Renal
Association guidelines (65\% of all patients commencing haemodialysis should commence with an AVF).

- Patients aged 75 or over were more likely to initiate RRT on an AVF (43.5\%) when compared to patients $<75$ years $(39.6 \%)$. Similarly, older patients were less likely to start on a tunnelled line $(31.5 \%$ vs. 39.6\%).

- Patients with polycystic kidney disease (PKD) as primary renal diagnosis were most likely to start on an AVF (67.4\%).

- Patients, who were referred at least 90 days prior to commencing dialysis, were more likely to start on an AVF compared to those starting more acutely (52.3\% vs. $5.5 \%)$.

- A high proportion of patients (33.6\%) who were referred at least 90 days prior to commencing dialysis, start dialysis on a tunnelled line.

- Patients who had been seen by a surgeon at least three months before starting dialysis were more likely to start on an AVF than those not assessed (72.4\% vs. $6.1 \%)$.

For PD:

- For a total of 1,021 first PD catheters, the insertion techniques were $38.3 \%$ open surgical, $13.3 \%$ laparoscopic, $3.2 \%$ peritoneoscopic and $28.0 \%$ 
Table 10.3. Patient characteristics stratified by type of first dialysis access

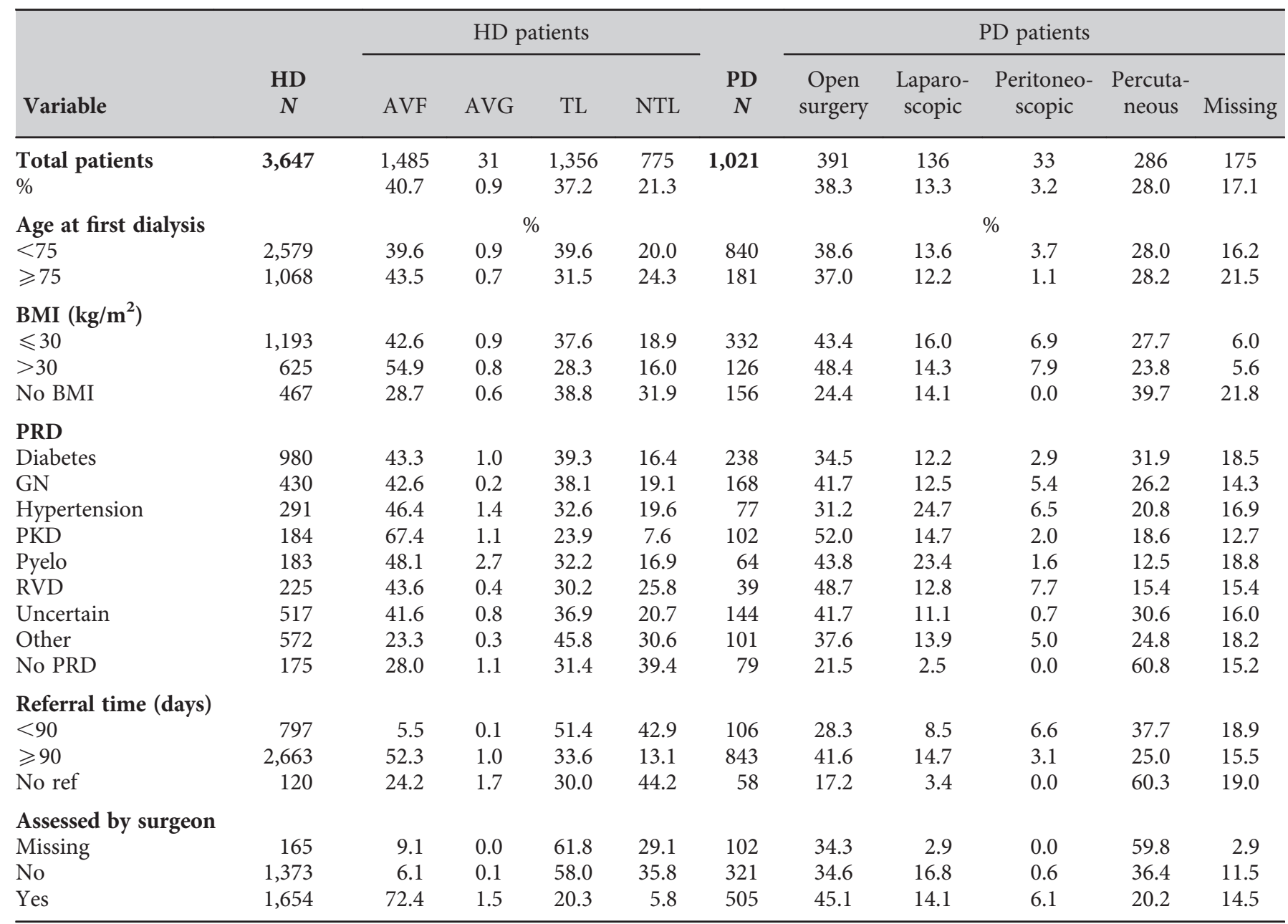

Patients from centres with more than $50 \%$ missing data for a variable are excluded from the table for that variable

$\mathrm{AVF}=$ arteriovenous fistula; $\mathrm{AVG}=$ arteriovenous graft; $\mathrm{TL}=$ tunnelled line; $\mathrm{NTL}=$ non-tunnelled line; $\mathrm{GN}=$ glomerulonephritis; $\mathrm{BMI}=$ body mass index; $\mathrm{PKD}=$ polycystic kidney disease; $\mathrm{PRD}=$ primary renal diagnosis; Pyelo = pyelonephritis; RVD = reno-vascular disease

percutaneous. Insertion technique was not reported for the remaining $17.1 \%$.

- Referral time had an influence on PD catheter insertion technique; $37.7 \%$ of patients referred less than 90 days before starting dialysis underwent percutaneous insertion compared to $25.0 \%$ of patients known longer to the service. These data were reversed for general surgical insertion: $28.3 \%$ of patients who presented late versus $41.6 \%$ of patients who did not present late.

- Patients who were assessed by a surgeon at least three months before starting dialysis were more likely to undergo open surgical placement $(45.1 \%$ vs. $34.6 \%$ for non-surgical assessment) and understandably less likely to undergo percutaneous catheter placement (20.2\% vs. $36.4 \%)$.
Figure 10.2 shows types of haemodialysis access stratified by age group. Patients aged less than 75 at the point of commencing RRT were less likely than older patients $(\geqslant 75)$ to start dialysis using an AVF $(39.6 \%$ vs. $43.5 \%)$ and more likely to start with a tunnelled line $(39.6 \%$ vs. $31.5 \%)$. The reason for this is unknown but may reflect patient engagement with renal services or varying progression of chronic kidney disease in the older population.

Figure 10.3 shows haemodialysis access stratified by PRD. The proportional distribution of HD access modality was similar for different primary renal disease diagnoses. Of note, patients with polycystic kidney disease were more likely to start HD on an AVF. This likely results from the opportunity for timely access preparation as these patients are often known to renal 


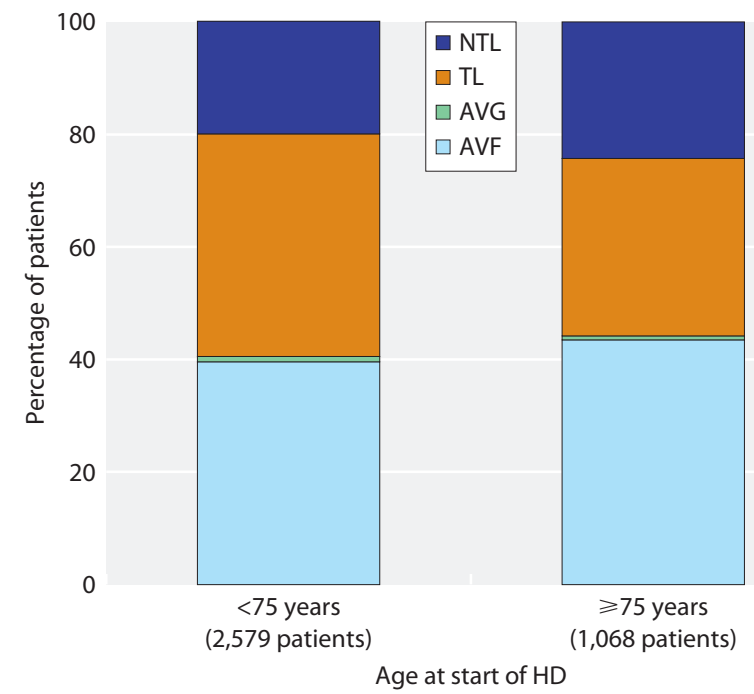

Fig. 10.2. Type of haemodialysis access stratified by age group $\mathrm{AVF}=$ arteriovenous fistula; $\mathrm{AVG}=$ arteriovenous graft; $\mathrm{TL}=$ tunnelled line; NTL $=$ non-tunnelled line

services for many years before dialysis is required. Where no primary renal diagnosis was available, the numbers of patients starting dialysis with a tunnelled or nontunnelled dialysis venous catheter were higher, suggesting that this may represent a cohort of patients who present late to renal services or whose renal function declines more rapidly than predicted.

Figure 10.4 shows PD catheter insertion methods stratified by BMI. Patients with body mass index (BMI) $>30 \mathrm{~kg} / \mathrm{m}^{2}$ were more likely to undergo open surgical placement $(51.3 \%)$ than those with BMI $\leqslant 30 \mathrm{~kg} / \mathrm{m}^{2}$

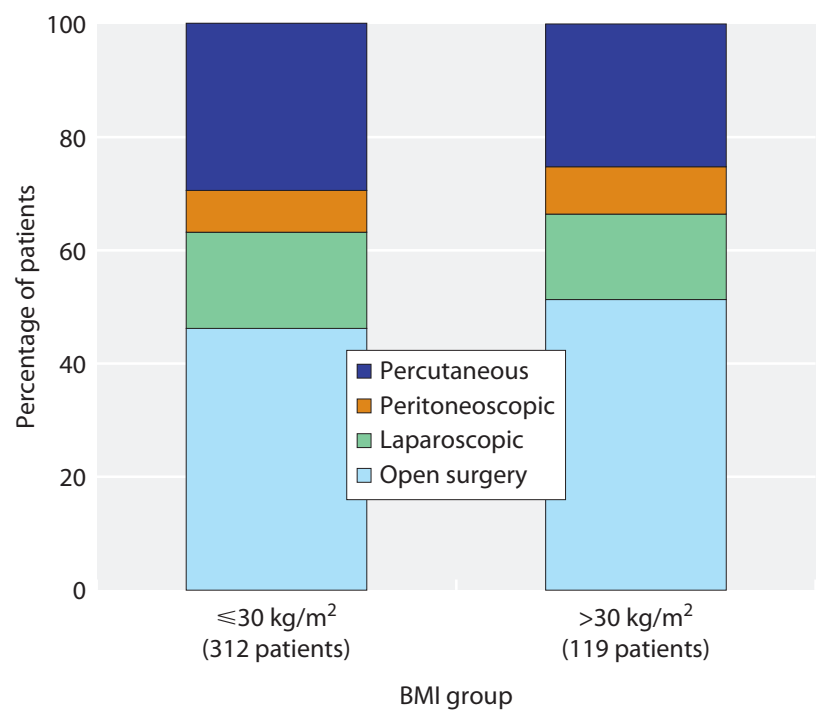

Fig. 10.4. Method of $\mathrm{PD}$ catheter insertion stratified by body mass index

$\mathrm{BMI}=$ body mass index

All patients from centres with more than $50 \%$ missing data for BMI were excluded

(46.2\%). The percutaneous approach was less likely to be used in patients in the higher BMI category (25.2\%) compared with those with a lower BMI (29.5\%). The peritoneoscopic or laparoscopic approach was used in a similar proportion of patients in both BMI groups. It should be noted that the analysis was limited due to a high proportion of missing data for BMI.

Figure 10.5 shows PD catheter insertion technique by centre. Centres reporting less than five patients on $\mathrm{PD}$ were not considered for analysis $(n=7)$. Eleven centres

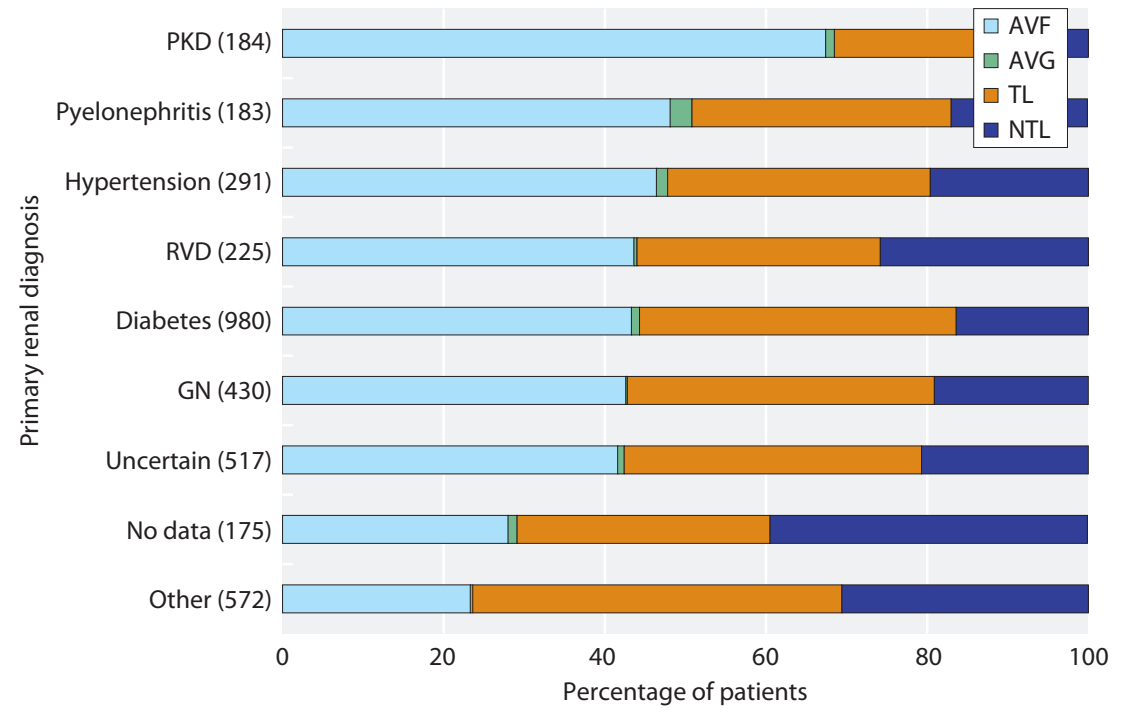

Fig. 10.3. Type of haemodialysis access stratified by primary renal disease Number of patients in each primary renal diagnosis group in brackets $\mathrm{AVF}=$ arteriovenous fistula; AVG $=$ arteriovenous graft; $\mathrm{TL}=$ tunnelled line; NTL = non-tunnelled line; $\mathrm{GN}=$ glomerulonephritis; RVD = renovascular disease; $\mathrm{PKD}=$ polycystic kidney disease 


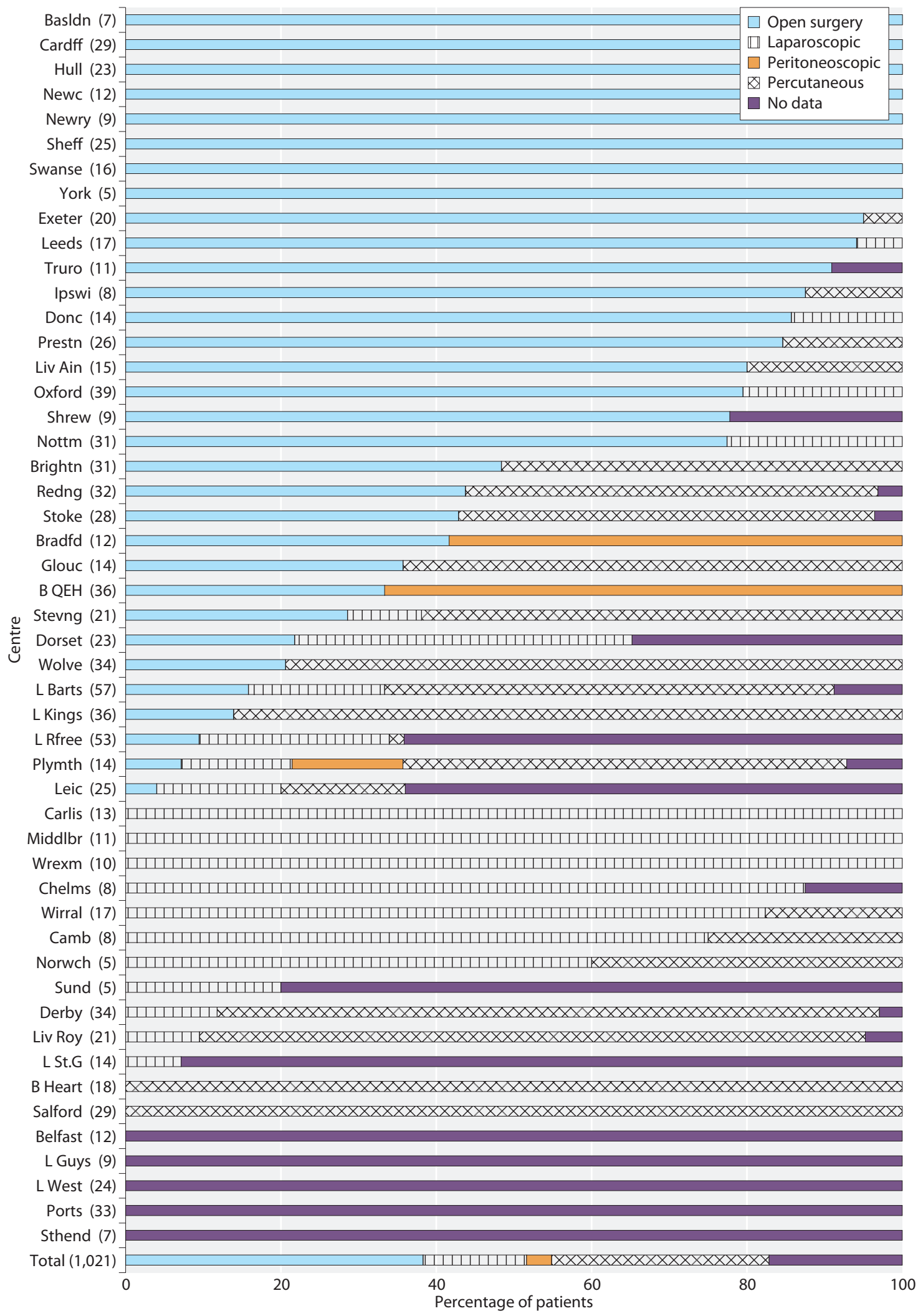

Fig. 10.5. PD catheter insertion technique stratified by centre 
reported less than 10 patients using PD catheters for first dialysis in 2013. There continues to be a strong tendency for many centres to rely on one single approach to $\mathrm{PD}$ catheter placement, with 13 centres reporting use of a single technique for all of their patients. Twenty-two centres reported using the percutaneous technique. Amongst these centres were some of those with the highest proportion of patients using a PD catheter as first access (Derby 45.9\%, London St Bartholemew's 39.5\%, Wolverhampton $37.3 \%$, Liverpool Roy $36.2 \%$, to name a few).

\section{First dialysis access and renal centre}

Figure 10.6 shows type of first dialysis access by centre. Variations were apparent between centres when considering patients commencing dialysis via an AVF, ranging from 10\% (London West, London St Bartholemew's, Wrexham) to 50\% (Birmingham Heartlands, Cambridge, Colchester, Liverpool Aintree). Some centres had over $60 \%$ of patients starting dialysis on a tunnelled line (London West, Sunderland, Dudley). The use of arteriovenous graft as the first dialysis access was between $0-5 \%$ with only 17 of the 57 centres opting to use this.

Use of a PD catheter as first access varied between $45 \%$ (Derby) and 0\% (Colchester, Clwyd, Dudley). In last year's audit there was some evidence that centres that had high usage of AVFs as starting access were also more likely to start patients on a PD catheter. Based on this year's audit, there is no evidence of this association.

The Renal Association guidelines on vascular access for Haemodialysis recommends 65\% of all patients commencing haemodialysis should commence with an AV fistula. This is depicted in figure 10.7 with patients who presented late excluded for this analysis. There were 14 centres below 2 standard deviations and a further 10 centres below 3 standard deviations. There were two centres above 2 standard deviations (Derby, Liverpool Aintree). The results have to be cautiously interpreted due to non-adjustment for any patient related factors. This may indicate variation in local processes for access planning and delivery and needs further investigation.

\section{First dialysis access and referral time}

Figure 10.8 shows a clear association between time known to a nephrologist and patient starting haemodialysis with an AVF. A greater proportion of patients who were known to a nephrologist for over one year started dialysis with an AVF, as compared to those who were referred between $90-365$ days ( $42.5 \%$ vs. $25.3 \%$ ). Similarly, patients who were known to a nephrologist between 90 days to over one year were more likely to start on PD when compared to patients who were referred $<90$ days prior to dialysis start.

Figure 10.9 shows PD catheter insertion technique by referral time. Patients who were first seen by a nephrologist $<90$ days before starting RRT were more likely to undergo percutaneous insertion when compared to patients who were known between 90-365 days and $>365$ days. Open surgical technique was less likely to be used in the patients presenting late when compared to the patients who were known over three months, probably because of having a lesser likelihood of seeing a surgeon.

Figure 10.10 shows first access for centres providing data for patients presenting late (known to renal services for $<90$ days). Amongst the 913 patients for whom data were reported, $45.3 \%$ started dialysis on a tunnelled line, $38.1 \%$ on a non-tunnelled line, $11.6 \%$ using a PD catheter with only $4.8 \%$ having first access documented as an AVF. There was however, wide variation amongst centres and clearly an understanding of practice patterns could lead to potential improvements in access service provision. There may also be reporting differences which need to be explored.

In 13 centres, more than $15 \%$ of patients presenting late had a peritoneal dialysis catheter inserted for use as first dialysis access and as a result had a lower requirement for tunnelled or non-tunnelled lines. However, the number of patients presenting late reported in some centres was extremely small and it is difficult to make firm observations about clinical pathways for the development of dialysis access in this cohort.

Figure 10.11 shows the type of haemodialysis access in patients known to the renal service for longer than three months. There was significant variation for patients starting haemodialysis on AVF, with Derby on one end of the spectrum at $91.6 \%$ and London West at the other end at $15 \%$. The centres with highest tunnelled line use were London West (76\%), Carlisle (73\%), London St Bartholemew's (67\%). There were five centres who reported over $30 \%$ of patients as starting on non-tunnelled lines despite being known to the centre for at least 90 days (Antrim 44.4\%, Shrewsbury 41.6\%, London Kings 37.7\%, London St Georges 50.0\%, Portsmouth 32.9\%). It will be important to understand the variations in practice patterns that lie behind these statistics which were not provided by current data.

\section{First dialysis access and surgical assessment}

Figure 10.12 highlights the proportion of patients who had been referred for surgical assessment at least three months prior to starting dialysis. There was considerable 


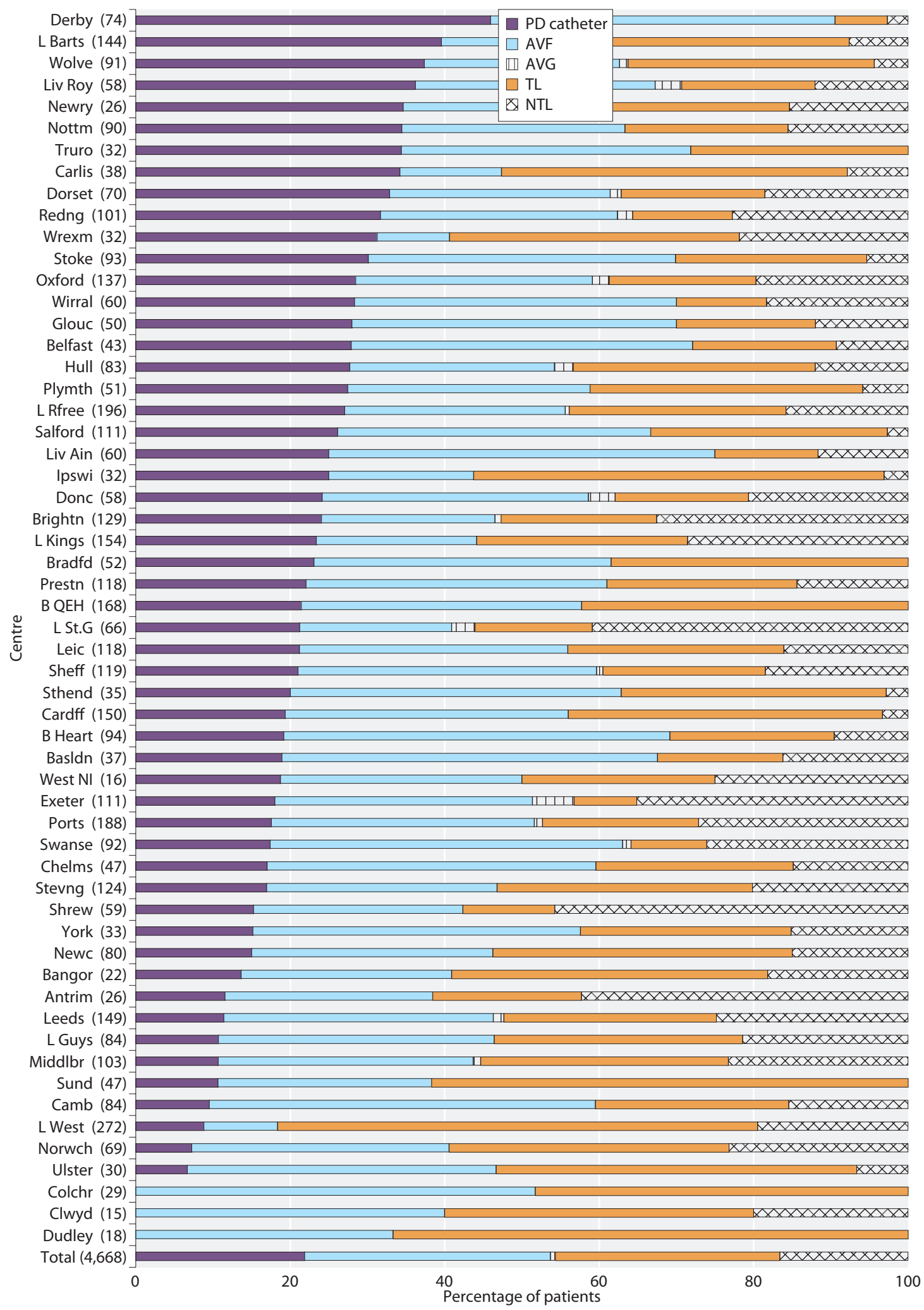

Fig. 10.6. Type of first dialysis access stratified by centre

$\mathrm{PD}=$ peritoneal dialysis; $\mathrm{AVF}=$ arteriovenous fistula; $\mathrm{AVG}=$ arteriovenous graft; $\mathrm{TL}=$ tunnelled line; $\mathrm{NTL}=$ non-tunnelled line 

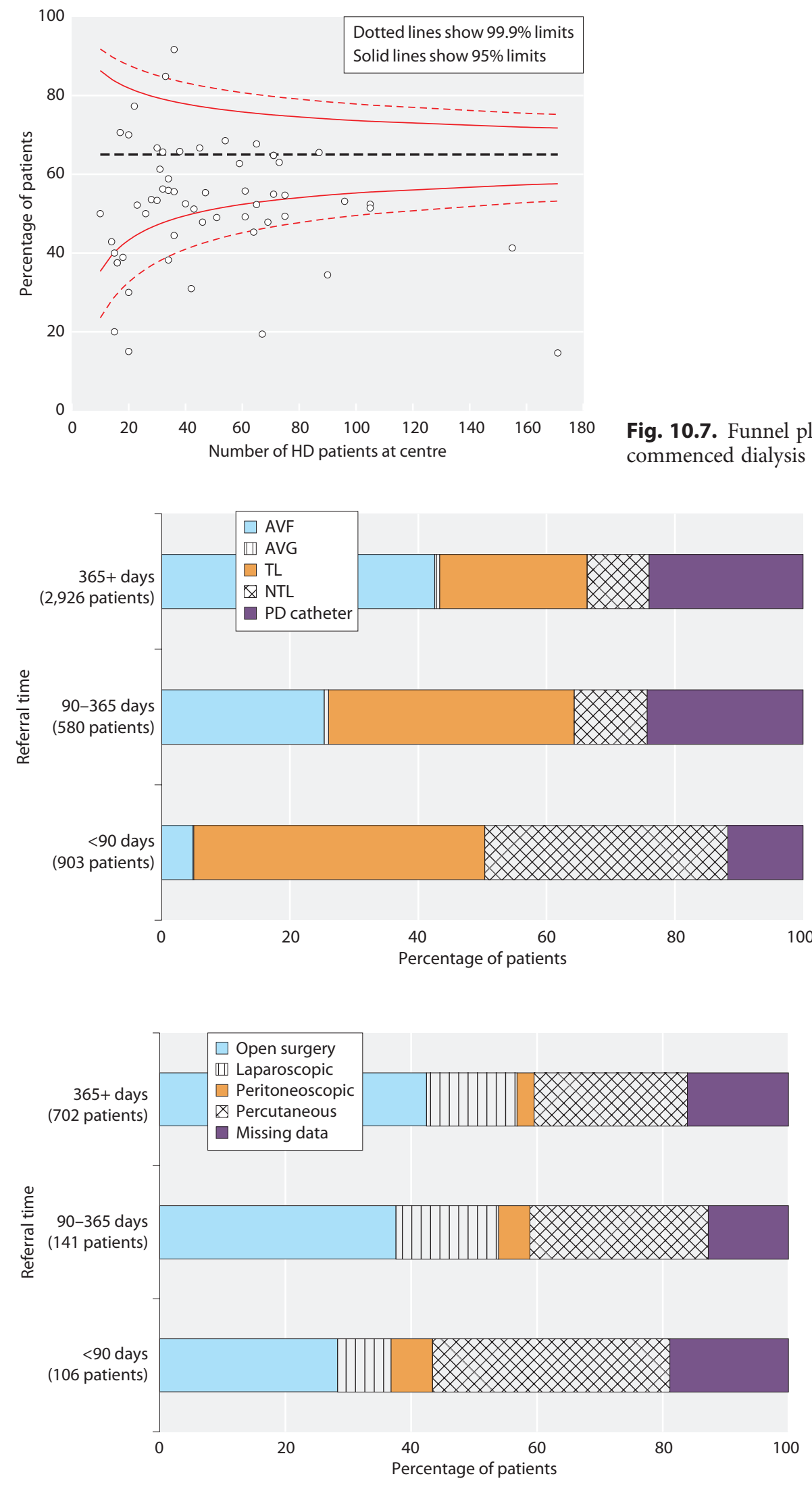

Fig. 10.7. Funnel plot of the percentage of HD patients who commenced dialysis using an AVF
Fig. 10.8. Type of first dialysis access by referral time
Fig. 10.9. PD catheter insertion technique by referral time 


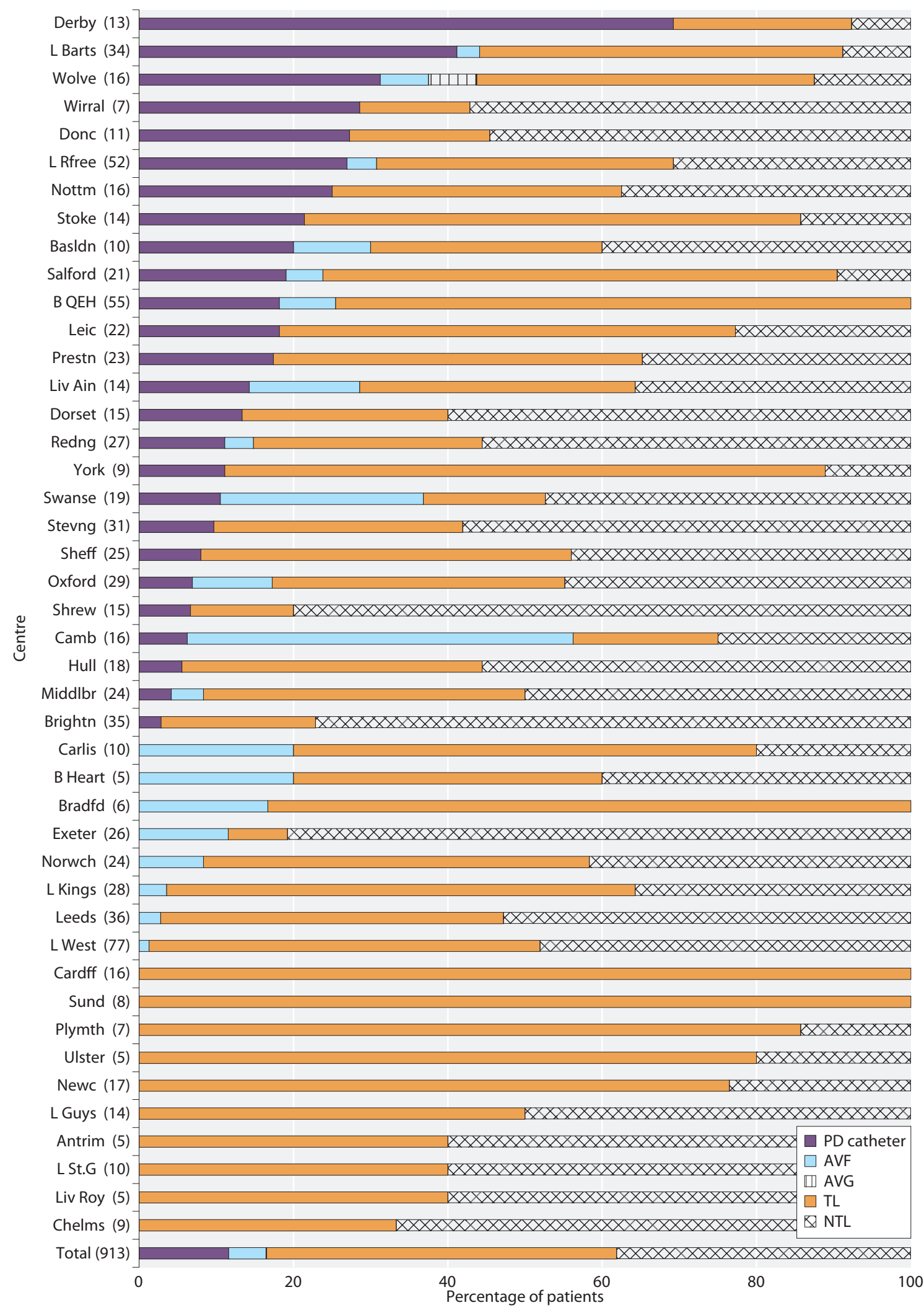

Fig. 10.10. Type of access used for first dialysis in patients presenting to a nephrologist $<90$ days prior to dialysis start $\mathrm{PD}=$ peritoneal dialysis; $\mathrm{AVF}=$ arteriovenous fistula; $\mathrm{AVG}=$ arteriovenous graft; $\mathrm{TL}=$ tunnelled line; $\mathrm{NTL}=$ non-tunnelled line 


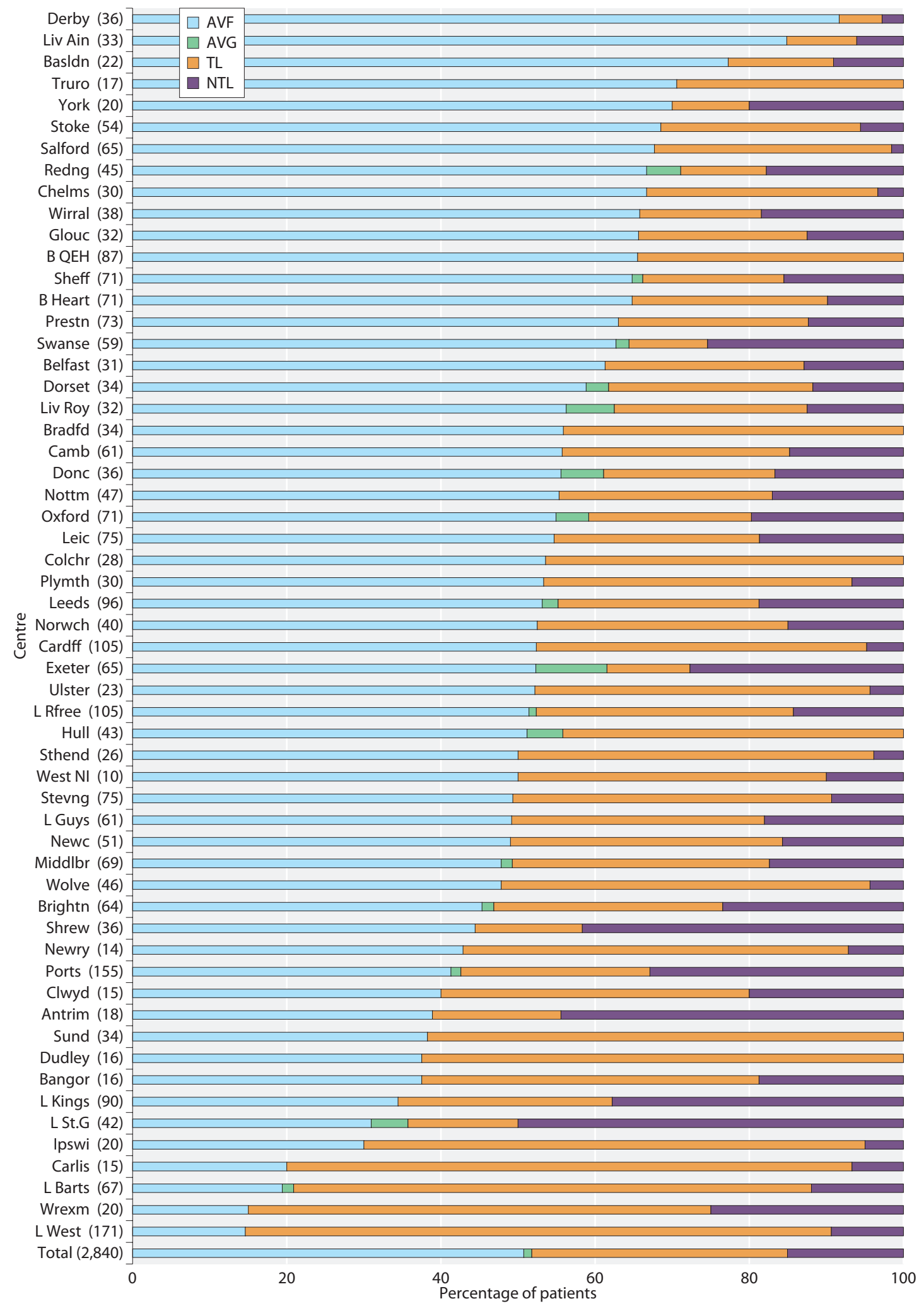

Fig. 10.11. Type of first access for haemodialysis stratified by centre restricted to patients presenting to a nephrologist $\geqslant 90$ days prior to start

$\mathrm{AVF}=$ arteriovenous fistula; $\mathrm{AVG}=$ arteriovenous graft; $\mathrm{TL}=$ tunnelled line; $\mathrm{NTL}=$ non-tunnelled line 


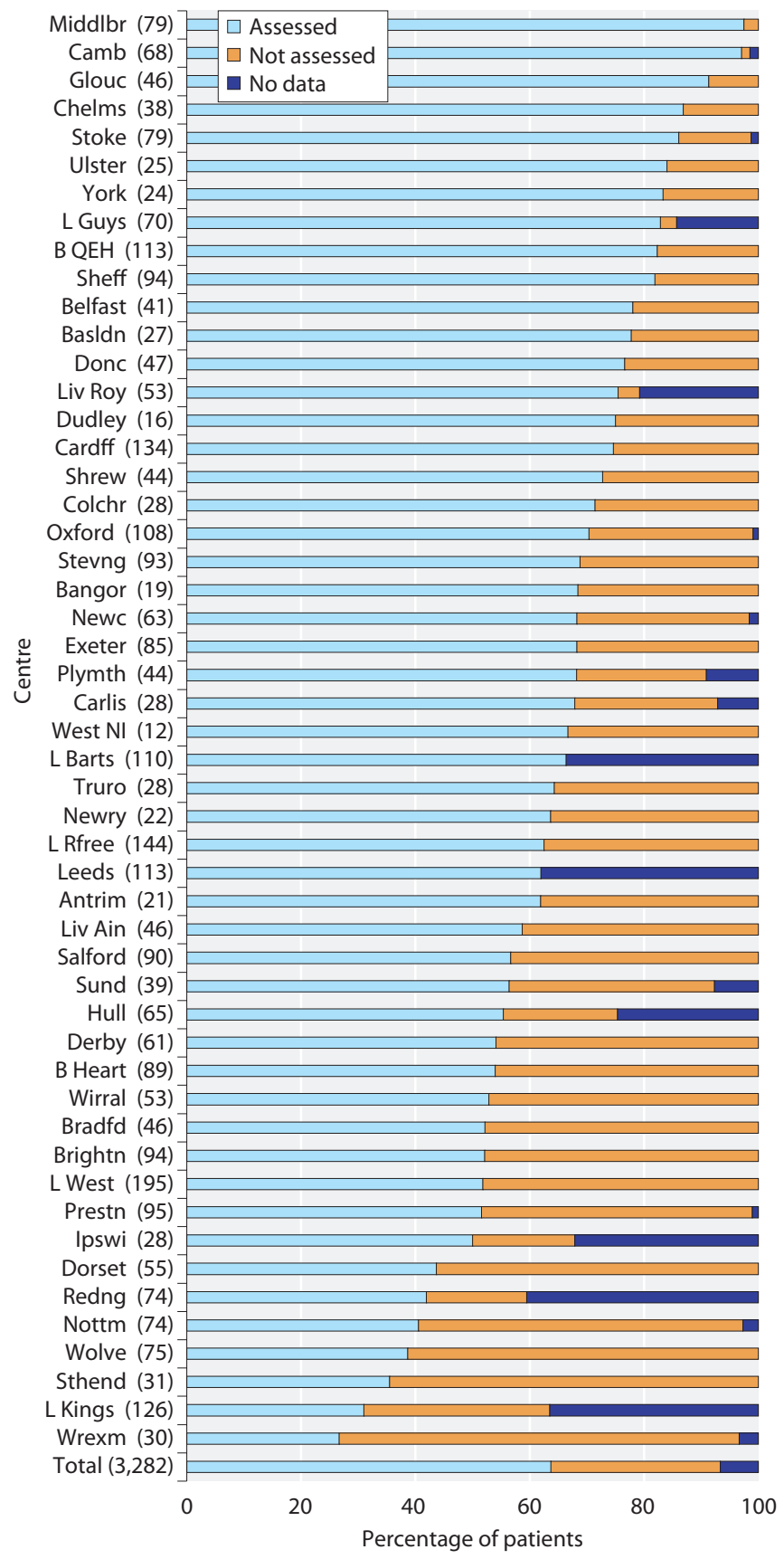

Fig. 10.12. Frequency of surgical assessment more than three months prior to starting dialysis

variation between the renal centres. Overall, the proportion referred to a surgeon was highest in Middlesbrough (97.5\%), Cambridge (97\%) and Gloucester (91.3\%). A detailed understanding of factors that prevent patients from being assessed for access in a timely fashion is required. These may reflect organisational factors or clinical uncertainty around the need for dialysis.

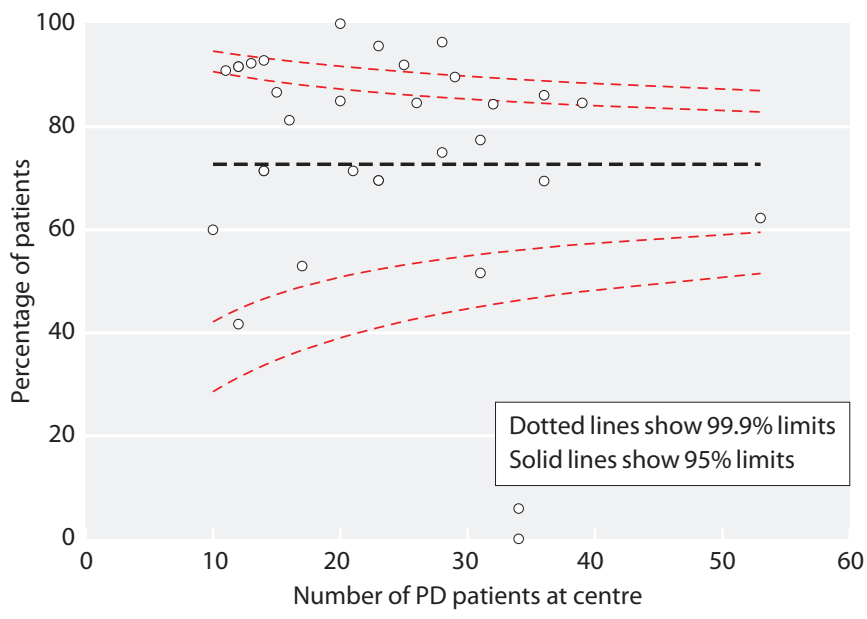

Fig. 10.13. Funnel plot of the percentage of patients with $P D$ catheter insertion $>2$ weeks before starting dialysis

Renal Association Peritoneal Access Clinical Guidelines state that $[4]$ :

'Whenever possible, catheter insertion should be performed at least 2 weeks before starting peritoneal dialysis. Small dialysate volumes in the supine position can be used if dialysis is required earlier.'

Figure 10.13 shows the variation in centres according to whether PD catheters were inserted at least two weeks prior to commencing dialysis. Thirty of the 32 centres had over $40 \%$ of patients with their PD catheters inserted at least two weeks prior to commencement of dialysis. Nottingham, Belfast, Derby and Wolverhampton were 2 standard deviations below the mean, probably because these centres carry out PD catheter insertion in late presenters. This guideline was intended to reduce the risk of dialysate leakage following catheter insertion, however it may actually have resulted in patients being less likely to use the PD catheter for early start PD and therefore possibly be exposed to the hazards of a central venous line. It is quite possible that this guideline has been a disincentive to using PD for patients presenting late or for acute kidney injury and revision should be considered in the next iteration of the guideline.

In the 2013 audit returns a greater proportion of patients who received surgical assessment at least three months prior to commencing dialysis underwent open surgical insertion ( $53.3 \%$ vs. $39.2 \%$ ) compared to those who did not (figure 10.14).

Figure 10.15 demonstrates a strong relationship between being assessed by a surgeon at least three months before starting dialysis and the likelihood of starting on an AVF. This relationship was much stronger than that 


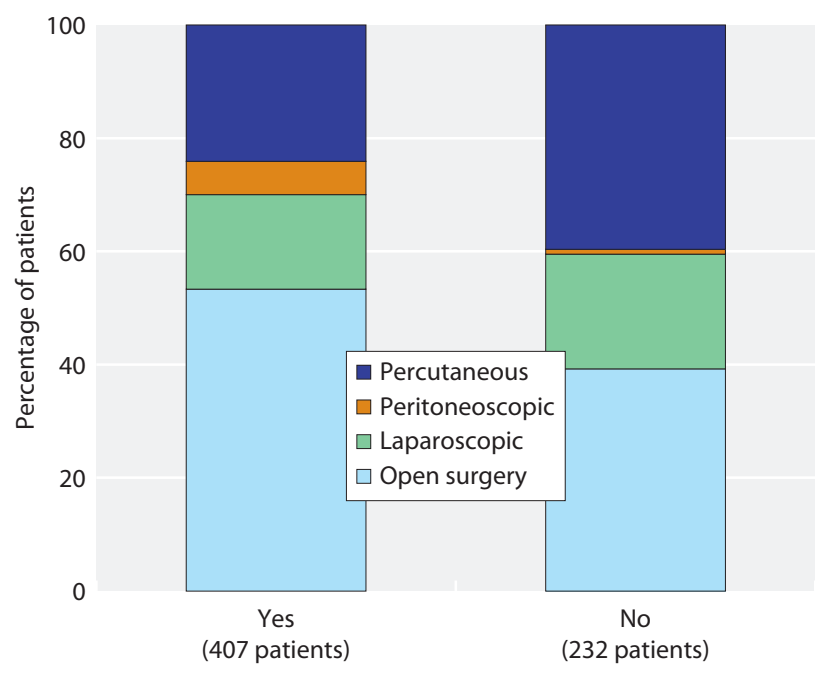

Assessment by surgeon at least three months before starting PD

Fig. 10.14. PD catheter insertion technique stratified by surgical assessment

between surgical assessment and method of PD catheter placement. This suggests that the role of surgical assessment was more important in relation to AVF placement. Of those assessed by a surgeon at least three months prior to starting dialysis, $73.5 \%$ started dialysis on an AVF whereas of those who were not seen by a surgeon only $10.1 \%$ did. Clearly, timely surgical assessment is a key component of the clinical pathway to fistula placement and without such assessment, patients are more likely to require temporary haemodialysis access such as a tunnelled or non-tunnelled dialysis catheter.

The relationship between surgical assessment and AVF formation was very different from that of PD catheter placement. It is quite possible that the time required to plan $\mathrm{PD}$ catheter placement is less than that required for AVF formation where vein mapping may be necessary.

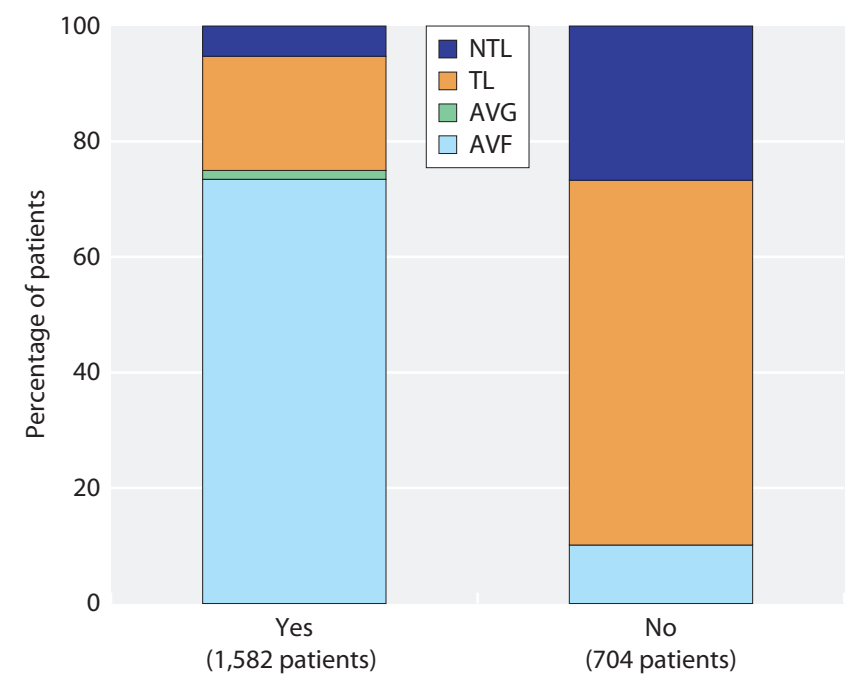

Assessed by a surgeon at least three months before starting dialysis

Fig. 10.15. Type of haemodialysis access stratified by surgical assessment

$\mathrm{AVF}=$ arteriovenous fistula; $\mathrm{AVG}=$ arteriovenous graft;

$\mathrm{TL}=$ tunnelled line; $\mathrm{NTL}=$ non-tunnelled line

\section{Dialysis access at three months after starting RRT}

The type of access used three months after starting dialysis gives an important insight into the responsiveness of the access formation pathway. Table 10.4 expresses the proportion of patients still dialysing using a particular form of access as a percentage of the access they originally started dialysis with. For example, $90.3 \%$ of patients starting dialysis with an AVF were still using this at three months and $88.4 \%$ of patients starting on PD remained on this modality at three months. Of patients starting dialysis via a tunnelled line, the majority continued to use this form of access at three months $(76.4 \%)$ and of 775 patients who commenced dialysis via a non-tunnelled line, 492 (63.6\%) were dialysing through a tunnelled line at three months. This may suggest that obtaining definitive access for HD within three months of starting treatment remains a challenge.

Table 10.4. Type of dialysis access at three months since dialysis start, stratified by first access type

\begin{tabular}{|c|c|c|c|c|c|c|c|c|c|}
\hline \multirow{2}{*}{$\begin{array}{l}\text { Access in use at } \\
\text { first dialysis }(N)\end{array}$} & \multicolumn{9}{|c|}{ Access in use at three months (\%) } \\
\hline & $\mathrm{AVF}$ & AVG & $\mathrm{TL}$ & NTL & PD catheter & Transplanted & Died & Stopped/LTFU & No data \\
\hline $\operatorname{AVF}(1,485)$ & 90.3 & 0.3 & 3.8 & 0.5 & 0.3 & 1.1 & 3.4 & 0.1 & 0.2 \\
\hline $\operatorname{AVG}(31)$ & 0.0 & 90.3 & 0.0 & 0.0 & 0.0 & 3.2 & 6.5 & 0.0 & 0.0 \\
\hline TL $(1,356)$ & 12.9 & 0.3 & 76.4 & 0.1 & 2.9 & 1.3 & 5.3 & 0.2 & 0.7 \\
\hline
\end{tabular}

$\mathrm{AVF}=$ arteriovenous fistula; $\mathrm{AVG}=$ arteriovenous graft; $\mathrm{TL}=$ tunnelled line; $\mathrm{NTL}=$ non-tunnelled line; $\mathrm{PD}=$ peritoneal dialysis; LTFU $=$ lost to follow up 
Figure 10.16 demonstrates the differences in access outcomes stratified by centre. By three months, $36 \%$ of patients were dialysing using an AVF (range 10\% London West to $67 \%$ Colchester); $0.9 \%$ were using an AVG (0\% many sites to $10 \%$ Exeter); $37 \%$ tunnelled lines (4\% Liverpool Aintree to $79 \%$ London West); 3\% nontunnelled lines; and $23 \%$ were using a PD catheter $(0 \%$ Colchester, Basildon, Dudley to 44\% Derby).

Access at 3 months in patients referred to renal centres $<90$ days before starting dialysis was analysed. Only 41 centres were included in this analysis. The majority (71\%) of patients presenting late were being dialysed using tunnelled lines at three months after dialysis start (figure 10.17). The between centre range was from $8 \%$ in Derby to $100 \%$ at Chelmsford and Sunderland. Amongst patients presenting late, only $11 \%$ were using an AVF at three months (individual centres ranged from $0 \%$ in nine centres to $42 \%$ in Exeter). PD catheters were used by $14 \%$ of patients (range $0 \%$ in 12 centres to $67 \%$ in Derby). It is interesting to note that in some centres late presentation is not always associated with poor start to a patient's dialysis pathway. These percentages must be interpreted with caution as reported numbers of patients presenting late tended to be low in many centres. Also further investigation is needed to review if patients who are dialysed via a tunnelled line at three months have a worse outcome compared to their counterparts who dialyse via AVF.

Figure 10.18 shows access in use at start of dialysis and at three months after commencing dialysis, displayed for all patients and also restricted to patients presenting late. There was a small increase in the proportion of patients dialysing with an AVF at three months for all patients (31.8\% to $36.1 \%)$. The increase was higher in the late presenters from $4.8 \%$ to $11.2 \%$. Use of a tunnelled line increased at three months in all patients $(8.4 \%)$ and in late presenters $(25.6 \%)$ respectively. This is clearly as a result of conversion from non-tunnelled line to tunnelled line. PD catheter use saw only a small increase for all patients (1.0\%) and for late presenters (2.6\%).

Figure 10.19 shows that the use of an AV fistula as the incident access was static between 2011 and 2013, with the proportion reported as roughly $38 \%$ in each year. Use of an AV graft has fallen from $1.4 \%$ to $0.8 \%$ over the three year period. Reported use of a tunnelled line, non-tunnelled line and peritoneal dialysis catheter has been static.

\section{Prevalent access}

Five centres were excluded from the analysis as the reported prevalent access numbers did not match with the number of prevalent patients at each of the centres in the UKRR database.

The Renal Association guidelines on vascular access for haemodialysis recommends $85 \%$ of all prevalent patients on haemodialysis should dialyse using an AV fistula. Twenty-six centres were more than three standard deviations and five centres were more than two standard deviations below this target (figure 10.20). The results have to be again cautiously interpreted due to nonadjustment for any patient related factors.

Figure 10.21 shows type of dialysis access in prevalent patients by centre. Variations were apparent between centres when considering prevalent patients with an $\mathrm{AV}$ fistula, ranging from about 40\% (Carlisle, Ipswich, London St Bartholemew's) to over 75\% (Cambridge, Birmingham Heartlands, Truro). Three centres had over $40 \%$ of prevalent patients on a tunnelled or non-tunnelled line (Ulster, Ipswich, Belfast) with two centres (Derby, Truro) at the other end of the spectrum with less than $10 \%$ of patients. The use of an AV graft was between $0 \%$ and $12 \%$ with 35 centres opting to use this.

Use of a PD catheter in prevalent patients varied between $28 \%$ at Carlisle and 3\% at Ulster (Colchester does not have any PD patients).

The percentage of prevalent patients using each type of dialysis access has not changed in the three years that the access audits have been collected (figure 10.22). Use of an AV fistula has been roughly $60 \%$ in each audit and use of PD has been approximately $15 \%$.

\section{Access failure}

Figure 10.23 shows comparative access failure for the different access types within three months. Access failure was defined as a documented date of failure/discontinuation recorded within three months of starting dialysis unless a centre comment indicated that it was a planned discontinuation. But there were deficiencies in the way that failure was recorded in this audit. Failure rates were generally higher in the peritoneal dialysis group with failure rates for percutaneous and open surgery at $10.5 \%$ and $8.2 \%$ respectively. Failure rates were generally around 5\% for haemodialysis access.

The number of access failures reported was small, however it can be seen from figure 10.24 that there was relatively poor reporting of the reason for failures. This may reflect the local documentation procedure. Infectious causes were reported as contributing to $10 \%$ of access failures of tunnelled lines and $0 \%$ for other haemodialysis accesses, and mechanical cause was reported as contributing to $90 \%$ of AVF failures. 


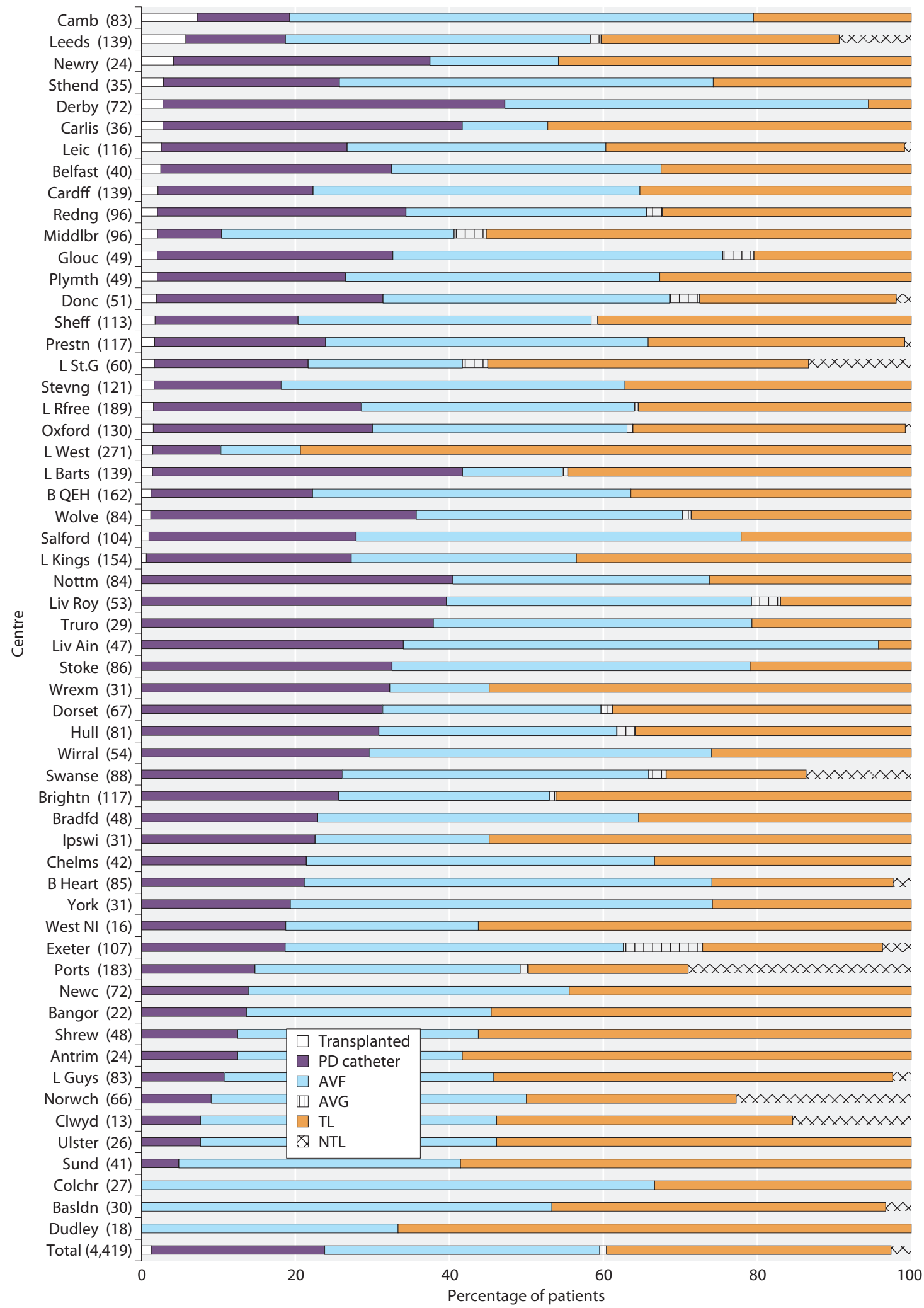

Fig. 10.16. Type of access at three months stratified by centre

$\mathrm{AVF}=$ arteriovenous fistula; $\mathrm{AVG}=$ arteriovenous graft; $\mathrm{TL}=$ tunnelled line; $\mathrm{NTL}=$ non-tunnelled line; $\mathrm{PD}=$ peritoneal dialysis 


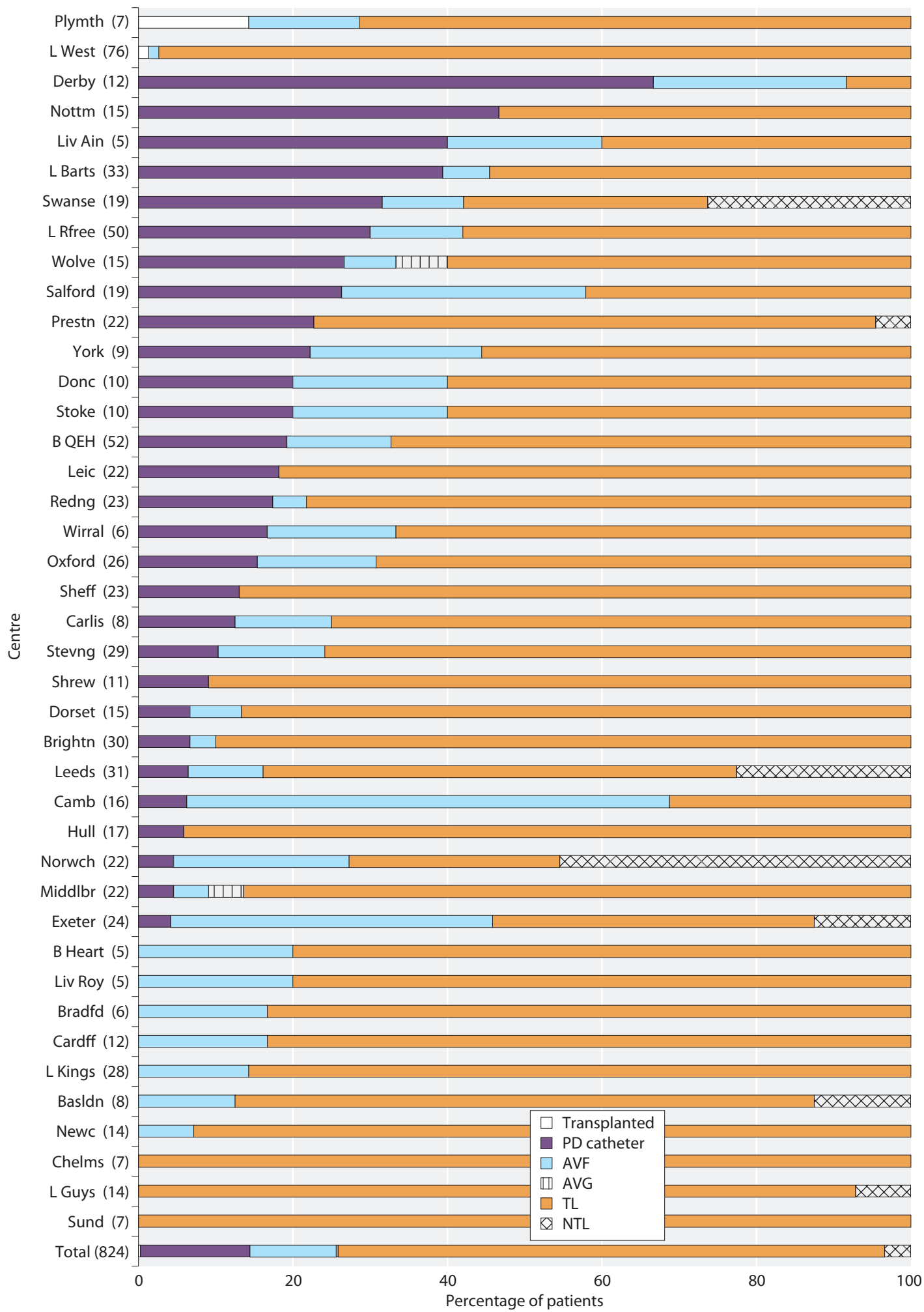

Fig. 10.17. Type of dialysis access at three months in patients referred to renal services $<90$ days before starting dialysis, stratified by centre

Centres reporting on fewer than five patients were excluded

$\mathrm{AVF}=$ arteriovenous fistula; $\mathrm{AVG}=$ arteriovenous graft; $\mathrm{TL}=$ tunnelled line; $\mathrm{NTL}=$ non-tunnelled line; $\mathrm{PD}=$ peritoneal dialysis 

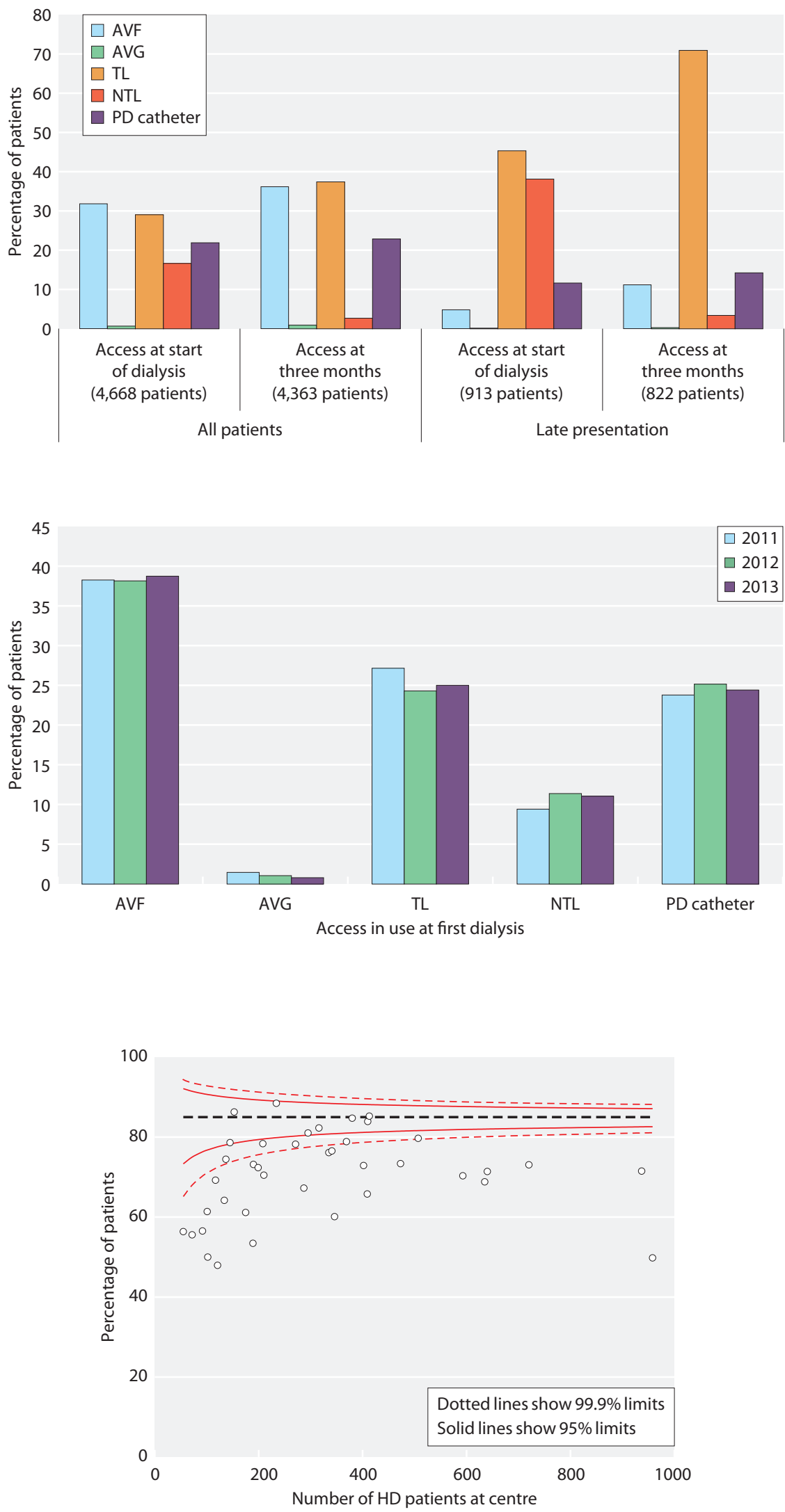

Fig. 10.18. Access in use at start of dialysis and after 3 months for those still on dialysis, displayed for all patients and also restricted to patients presenting late $\mathrm{AVF}=$ arteriovenous fistula; $\mathrm{AVG}=$ arteriovenous graft; $\mathrm{TL}=$ tunnelled line; NTL = non-tunnelled line; $\mathrm{PD}=$ peritoneal dialysis

Fig. 10.19. Percentage trend in incident dialysis access use at first dialysis $\mathrm{AVF}=$ arteriovenous fistula; $\mathrm{AVG}=$ arteriovenous graft; $\mathrm{TL}=$ tunnelled line; NTL = non-tunnelled line; $\mathrm{PD}=$ peritoneal dialysis

Fig. 10.20. Funnel plot of the percentage of prevalent $\mathrm{HD}$ patients dialysing using an AVF 


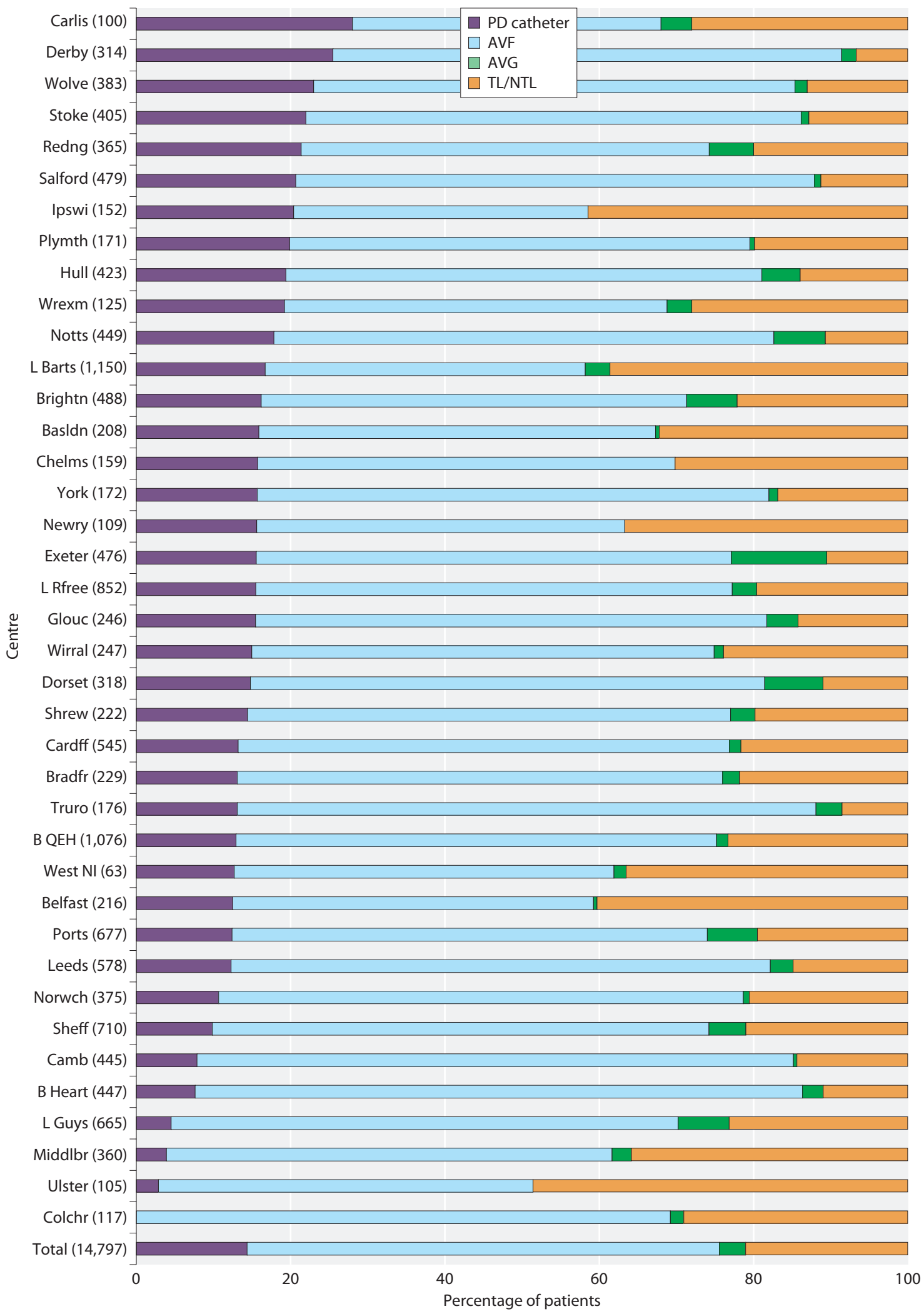

Fig. 10.21. Type of dialysis access in prevalent patients stratified by centre

$\mathrm{PD}=$ peritoneal dialysis; AVF = arteriovenous fistula; AVG = arteriovenous graft; $\mathrm{TL}=$ tunnelled line; NTL $=$ non-tunnelled line 

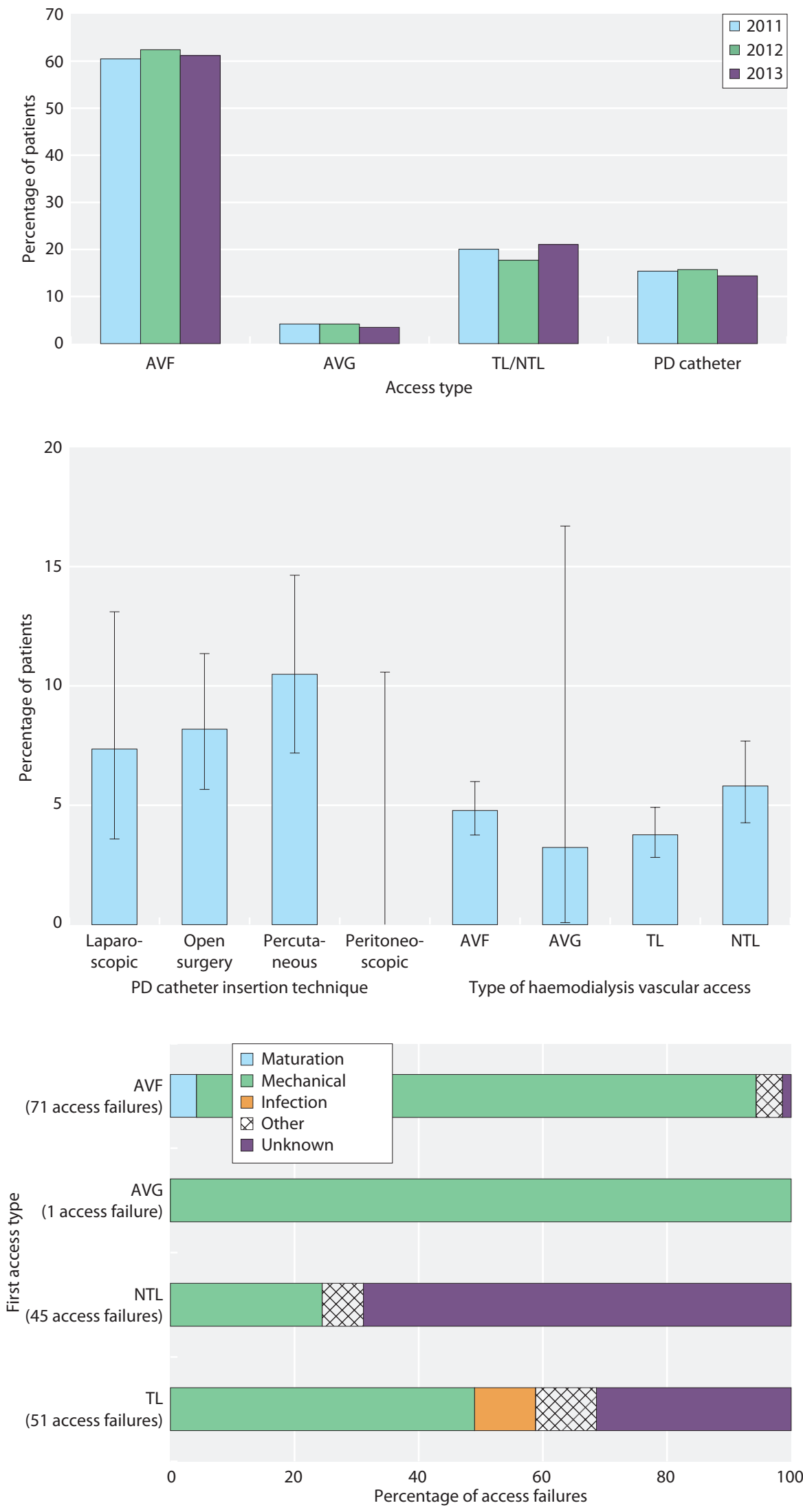

Fig. 10.22. Percentage of prevalent dialysis patients with each access type, by year

$\mathrm{AVF}=$ arteriovenous fistula;

$\mathrm{AVG}=$ arteriovenous graft; $\mathrm{TL}=$ tunnelled line; NTL $=$ non-tunnelled line; $\mathrm{PD}=$ peritoneal dialysis

Fig. 10.23. Percentage of patients experiencing failure of first access within three months, by type of first access $\mathrm{AVF}=$ arteriovenous fistula;

$\mathrm{AVG}=$ arteriovenous graft; $\mathrm{TL}=$ tunnelled line; NTL = non-tunnelled line; $\mathrm{PD}=$ peritoneal dialysis

Fig. 10.24. Reported causes of haemodialysis access failure within three months stratified by first access type $\mathrm{AVF}=$ arteriovenous fistula;

$\mathrm{AVG}=$ arteriovenous graft; $\mathrm{TL}=$ tunnelled line; NTL = non-tunnelled line

Rao/Pitcher/Fluck/Kumwenda 


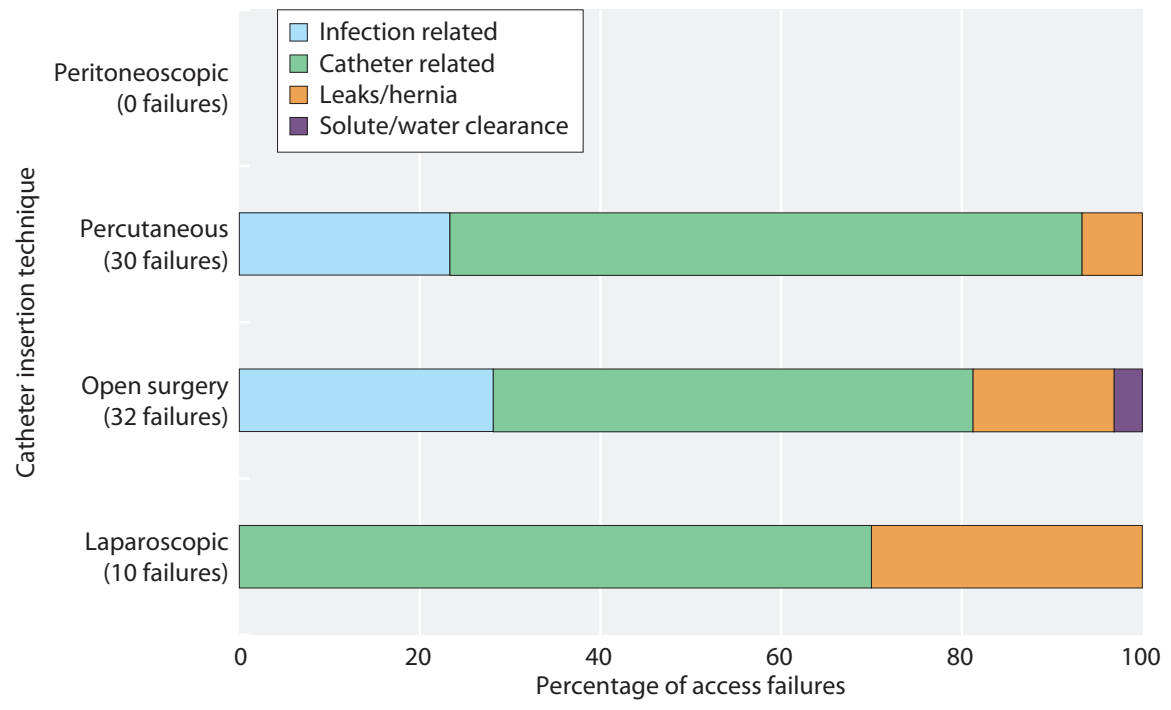

Fig. 10.25. Reported causes of peritoneal dialysis access failure within three months stratified by catheter insertion technique
Again, numbers of PD access failure were small and hence drawing any inferences is difficult. However, it can be seen from figure 10.25 that peritoneoscopic technique had no documented failures within three months. Catheter related cause ranged from $53 \%$ to $70 \%$ of PD failure for each of the three other listed insertion techniques. Failure associated with leaks or hernia was highest for laparoscopic insertion. Twenty-seven out of 832 (3.25\%) PD patients were reported as experiencing peritonitis within two weeks of catheter insertion (data not shown). This was significantly lower than the national target of $5 \%$.

\section{PD access audit one-year follow-up}

Centres who reported on PD patients in the 2012 vascular and peritoneal access audits were asked to complete a one year follow up of their PD patients. The additional information requested was the date of catheter failure, the reason for catheter failure, the number of catheters used during the year, and the modality in use at one year after starting PD. Of 50 centres who reported data on PD patients in 2012, 28 completed the one year follow up, returning data on 522 (56\%) patients.

In these patients, $290(55.5 \%)$ were still on PD at one year with $88.3 \%$ of these $(256 / 290)$ on their first catheter.

The majority of centres maintained $>50 \%$ of patients on PD at one year, however only four centres maintained $>80 \%$ on PD at one year. Modality change to haemodialysis varied from 0\% (Antrim, Newry, Doncaster) to 43\% (Sunderland, Chelmsford). For patients who started on PD in 2012, transplantation varied at centres from $0 \%$ to $55 \%$ (figure 10.26).
Causes of PD access failure within one year of starting on PD were analysed. The reported numbers were too low to draw firm conclusions. Unsurprisingly the principal causes of catheter failure were mechanical or infection related (figure 10.27).

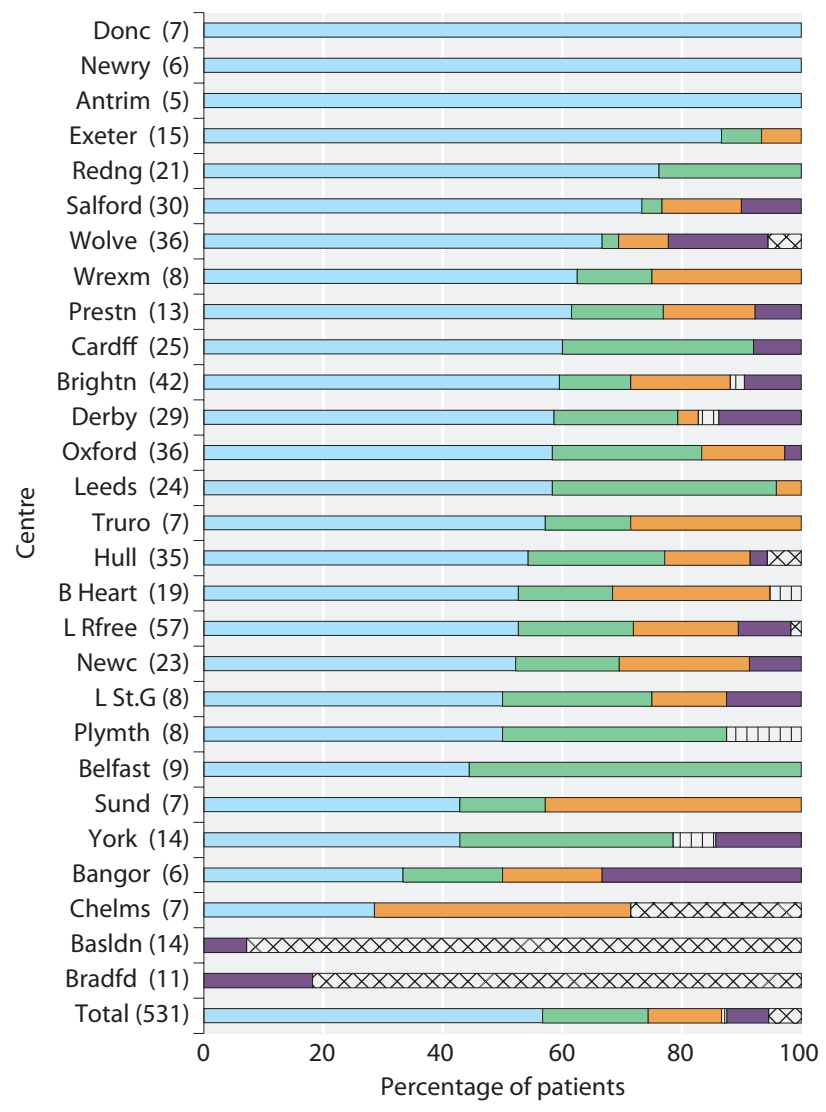

Fig. 10.26. Modality at one year after commencing PD, by centre 


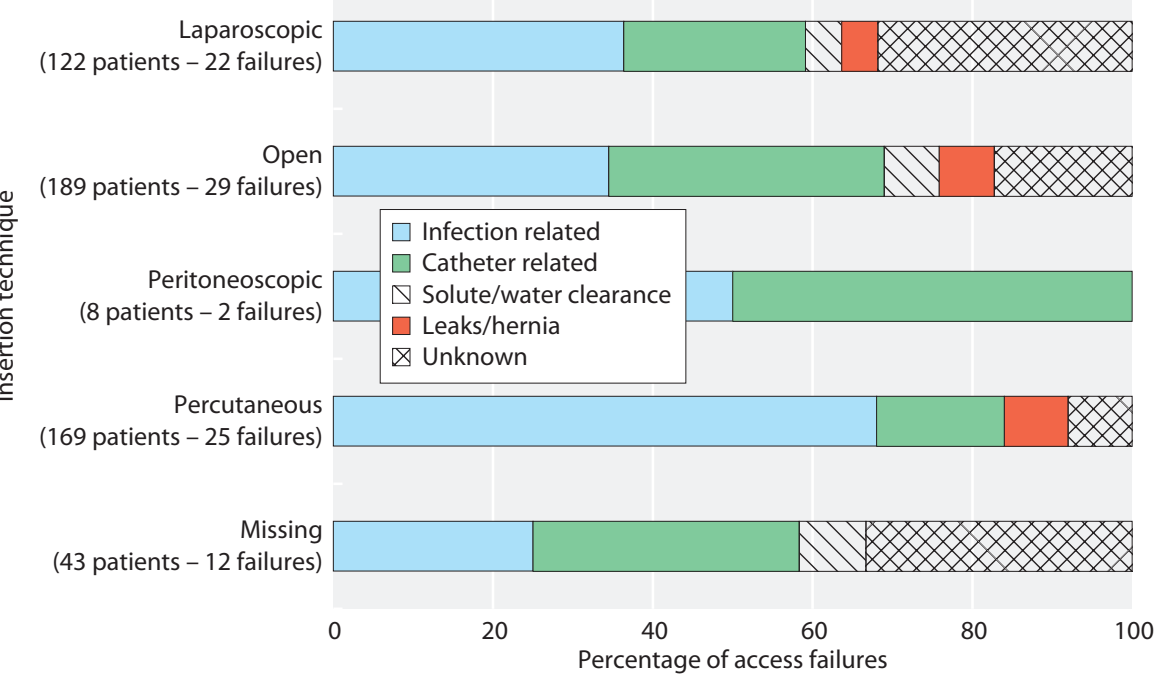

Fig. 10.27. Causes of PD access failure within one year of PD catheter insertion
Figure 10.28 is a funnel plot which graphically displays the unadjusted percentage of PD patients experiencing a catheter failure within one year of commencement of RRT across multiple renal centres. PD catheter failure was censored for transplantation, elective transfer to HD or death. The results have to be cautiously interpreted due to the extent of and variation in missing data, small numbers of patients in some centres and non-adjustment for any patient related factors.

Of the centres for which data were available $(n=17)$, no outlier centres were identified with failure rates above the upper $95 \%$ 'alert' or $99.9 \%$ 'alarm' limits for PD catheter failures. One renal centre reported one-year catheter failure rate below the $95 \%$ control limit. The

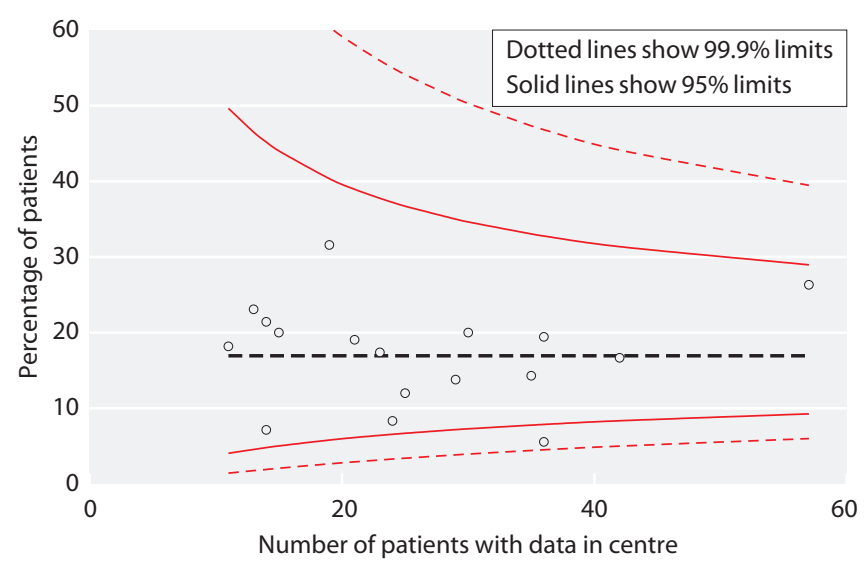

Fig. 10.28. Funnel plot of the percentage of PD catheter failures within one year of insertion mean one-year catheter failure rate was $17 \%$ which was below the rate recommended in the guidelines issued by the ISPD/RA (23\% and $20 \%$ respectively).

\section{Conclusions}

This second multisite dialysis access audit from England, Wales and Northern Ireland has provided important information regarding the variation in access provision and failure. Data collection is still not optimal as significant amounts of missing data across a range of fields exist. Several operational definitions need to be refined for further audit collections. As over a fifth of haemodialysis patients start dialysis on a non-tunnelled line, it may be preferable to collect dialysis access at the fourth week as well as the first dialysis session since both non-tunnelled and tunnelled catheters are often used before more permanent access is placed (PD catheter, AVG or AVF). Of concern is that tunnelled lines continued to be used in approximately a third of patients three months post dialysis start and this figure was higher for patients presenting late. The practice of PD catheter insertion in patients presenting late was used by relatively few centres. Only 13 out of 43 centres with sufficient data on patients presenting late placed a peritoneal dialysis catheter in more than $15 \%$ of patients as first dialysis access. It was also interesting to note that in some centres late presentation was not always associated with poor start to a patient's dialysis pathway. 
Surgical assessment was key to formation of permanent vascular access (AVF/AVG), 73\% started dialysis on an AVF whereas of those who were not seen by a surgeon only $10 \%$ did. Twenty-four centres were 2 or 3 standard deviations below the $65 \%$ target for incident patients starting haemodialysis on AVF and 31 centres were below the $85 \%$ target for prevalent haemodialysis patients on AVF. The vascular access clinical practice guidelines are due for review this year and the authors need to reconsider whether these current standards should be changed. Further analyses are being planned to explore why there is such a wide variation in access provision between centres and whether the type of vascular access in use at dialysis start explains the different outcomes.

\section{Acknowledgement}

Thanks are expressed to the Healthcare Quality Improvement Partnership (HQIP) who contributed to the funding of the PD access audit and all renal centres for their assistance in providing the data.

Conflicts of interest: none

\section{References}

1 Briggs V, et al.: UK Renal Registry 15th annual report: Chapter 8 UK multisite peritoneal dialysis access catheter audit for first PD catheters 2011. Nephron Clin Pract, 2013;123(Suppl 1):165-81

2 NHS Information Centre. National Kidney Care Audit Vascular Access Report: 2011 [cited 2013 1st October]. Available from: http://www.hqip. org.uk/assets/NCAPOP-Library/VAReport2011Interactive03082011FINAL.pdf
3 Fluck R, Kumwenda Dr M: Renal Association Vascular Access for Haemodialysis clinical guidelines, 2011. Available from: http://www. renal.org/guidelines/modules/vascular-access-for-haemodialysis\#sthash. 8TAwquhO.dpbs

4 Wilkie M, Jenkins S, Shrestha B: Renal Association Peritoneal dialysis access clinical guidelines. 2009. Available from: http://www.renal.org/ guidelines/modules/peritoneal-access\#sthash.xqAutugK.dpbs 